\title{
Nonlinear vibration of a continuum rotor with transverse electromagnetic and bearing excitations
}

\author{
Haiyang Luo ${ }^{\mathrm{a}, \mathrm{b}, *}$ and Yuefang Wang ${ }^{\mathrm{a}, \mathrm{b}}$ \\ ${ }^{a}$ State Key Laboratory of Structural Analysis for Industrial Equipment, Dalian, China \\ ${ }^{\mathrm{b}}$ Department of Engineering Mechanics, Dalian University of Technology, Dalian, China \\ Received 24 August 2011 \\ Revised 27 December 2011
}

\begin{abstract}
The nonlinear vibration of a rotor excited by transverse electromagnetic and oil-film forces is presented in this paper. The rotor-bearing system is modeled as a continuum beam which is loaded by a distributed electromagnetic load and is supported by two oil-film bearings. The governing equation of motion is derived and discretized as a group of ordinary differential equations using the Galerkin's method. The stability of the equilibrium of the rotor is analyzed with the Routh-Hurwitz criterion and the occurrence of the Andronov-Hopf bifurcation is pointed out. The approximate solution of periodic motion is obtained using the averaging method. The stability of steady response is analyzed and the amplitude-frequency curve of primary resonance is illustrated. The Runge-Kutta method is adopted to numerically solve transient response of the rotor-bearing system. Comparisons are made to present influences of electromagnetic load, oil-film force and both of them on the nonlinear vibration response. Bifurcation diagrams of the transverse motion versus rotation speed, electromagnetic parameter and bearing parameters are provided to show periodic motion, quasi-periodic motion and period-doubling bifurcations. Diagrams of time history, shaft orbit, the Poincaré section and fast Fourier transformation of the transverse vibration are presented for further understanding of the rotor response.
\end{abstract}

Keywords: Rotor, nonlinear vibration, electromagnetic load, oil-film force, stability, bifurcation

\section{Introduction}

It is well known that rotors of electric motors operate in electromagnetic fields distributed inside the clearance, called air-gap, between the stator and the rotor. Practically, the intensity of the electromagnetic field is not uniform in the circumferential direction of the air-gap due to dynamic deformation of the rotor shaft. Consequently, a resultant attraction load is generated on the rotor pointing outwardly to the shortest air-gap, this electromagnetic load is known as the unbalanced magnetic pull (UMP). This electromagnetic load may excite severe transverse vibrations and hence jeopardize the safety of the rotor. Previous researches on modeling the electromagnetic load on rotors have been published. Summers proposed a method for computing electromagnetic load using rotating magnetic field component [1]. Belmans et al. expressed the electromagnetic load in a two-pole induction motor considering the effect of homopolar flux and analyzed the double supply frequency of the load [2]. Smith and Dorrell obtained the electromagnetic load in cage induction motors based on theoretical and experimental investigations $[3,4]$. Bi and Liu studied the electromagnetic load in spindle motors of hard disk drives using magnetic circuit and field theories [5]. For electromagnetic-induced vibrations, the transverse motion of rotors related to electromagnetic loads has been investigated as well. Lundström and Aidanpää analyzed large eccentricities of the rotor of an

\footnotetext{
* Corresponding author: State Key Laboratory of Structural Analysis for Industrial Equipment, Dalian 116024, China. Tel.: +86 15840639767; Fax: +86411 84706571; E-mail: luohy1725@126.com.
} 
electric generator [6]. Belmans et al. investigated stability of flexible-shaft induction machines and showed excessive vibrations of rotors caused by electromagnetic load [7]. Guo et al. obtained the vibration of a model rotor in a threephase generator which is excited by electromagnetic load and mass unbalance [8]. The electro-mechanical interaction and nonlinear phenomenon of high-speed turbo-generators with smooth poles are presented by Pennacchi considering the electromagnetic load in air-gap [9]. Wang et al. derived the analytic expression of steady electromagnetic load and investigated the nonlinear vibration and stability of a Jeffcott rotor with unbalanced magnetic excitation [10]. Luo et al. used harmonic balance method and Runge-Kutta method to present the stability and bifurcation of a continuum rotor supported by short sliding bearings and excited by distributed electromagnetic load [11].

As far as nonlinear rotor dynamics is concerned, numerous researches have been published to present various types of periodic motions, super- and sub-harmonic motions, bifurcations and chaotic motions for practical rotor-bearingseal systems (cf. [12-19]). Nevertheless, it is worth pointing out that rotor systems were modeled as Jeffcott rotors in most of literature, which are not accurate for modeling the systems with longitudinally distributed excitations. Several models have been developed to investigate continuum rotor systems with oil-film forces and transverse excitations. Muszynska analyzed the stability of a model of lightly loaded rotating shaft-bearing-seal systems [20]. Azeez and Vakakis obtained the transient response of an overhung rotor undergoing vibro-impacts due to a defective bearing [21]. Oncescu et al. used the finite element formulation to investigate the stability and steady state response of a rotor with bearings and asymmetric shaft cross-section [22]. Jing and Meng et al. adopted the finite element method to solve nonlinear dynamics of a rotor-bearing system based on a continuum model [23]. Using the method of harmonic balance, Hosseini and Khadem studied the combination resonances of an inextensible, simply supported rotating shaft with nonlinearities in curvature and inertia [24].

In spite of the publications on general rotor-bearing systems and rotor systems under excitation of electromagnetic load, very few of them focused on continuum rotors with both of oil-film bearing and transverse electromagnetic load. In this paper, the nonlinear vibration of a bearing-supported rotor is presented with excitation of a steady, transverse electromagnetic load. The rotor-bearing system is modeled as an Euler-Bernoulli beam to admit the longitudinally distributed electromagnetic load and mass eccentricity. The governing equation of motion is derived and discretized as a group of ordinary differential equations using the Galerkin's method. The stability of the rotor's equilibrium is determined through the Routh-Hurwitz criterion for the linearized system. For forced vibrations caused by unbalanced mass, the amplitude of steady-state motion is solved approximately using the averaging method, and the jump phenomenon is pointed out with the curve of amplitude-frequency relation of primary resonance. The nonlinear transverse vibration of the rotor is obtained with the Runge-Kutta method, and how it varies with rotation speed, electromagnetic load and bearing parameters is shown through numerical examples. Comparisons are made to demonstrate the influence of electromagnetic load and bearing parameters on displacement response of the rotor. The complexity of the vibration response is shown through plots of bifurcation diagram, time history, shaft orbit, the Poincaré section and the fast Fourier transformation of the motion. Periodic motions, quasi-periodic motions and period-doubling bifurcations are found out based on the numerical analysis.

\section{Mathematical model}

A continuum rotor-bearing system loaded by a longitudinally distributed, transverse electromagnetic load is shown in Fig. 1, where $P_{m}$ denotes the electromagnetic load, $X_{a}$ and $X_{b}$ are locations of the two oil-film forces which are denoted by $F_{a}$ and $F_{b}$, respectively. Figure 2 shows the cross section of the rotor, where $O$ is the center of stator, $O^{\prime}$ is the center of shaft and $O_{C}$ is the mass center of the shaft. $\phi$ is the angle of the minimum air-gap. $e$ is the dynamic displacement of $O^{\prime}$ with respect to $O$.

The shaft is modeled as a uniform Euler-Bernoulli beam supported by two bearings in this study. The gyroscopic effects are ignored since the rotor is considered a slender beam which the ratio between the length of the beam and its diameter is more than 10 [25]. The governing equation of motion is developed as follows:

$$
\begin{aligned}
& {\left[\begin{array}{cc}
\bar{m} & 0 \\
0 & \bar{m}
\end{array}\right]\left(\begin{array}{l}
\partial^{2} Y / \partial t^{2} \\
\partial^{2} Z / \partial t^{2}
\end{array}\right)+E I\left(\begin{array}{c}
\partial^{4} Y / \partial X^{4} \\
\partial^{4} Z / \partial X^{4}
\end{array}\right)=\left(\begin{array}{l}
\bar{m} g \\
0
\end{array}\right)} \\
& +\bar{m} e_{\bar{m}} \omega^{2}\left(\begin{array}{c}
\cos \omega t \\
\sin \omega t
\end{array}\right)+P_{m}\left(\begin{array}{c}
\cos \phi \\
\sin \phi
\end{array}\right)+\delta\left(X-X_{a}\right)\left(\begin{array}{c}
F_{a Y} \\
F_{a Z}
\end{array}\right)+\delta\left(X-X_{b}\right)\left(\begin{array}{l}
F_{b Y} \\
F_{b Z}
\end{array}\right)
\end{aligned}
$$




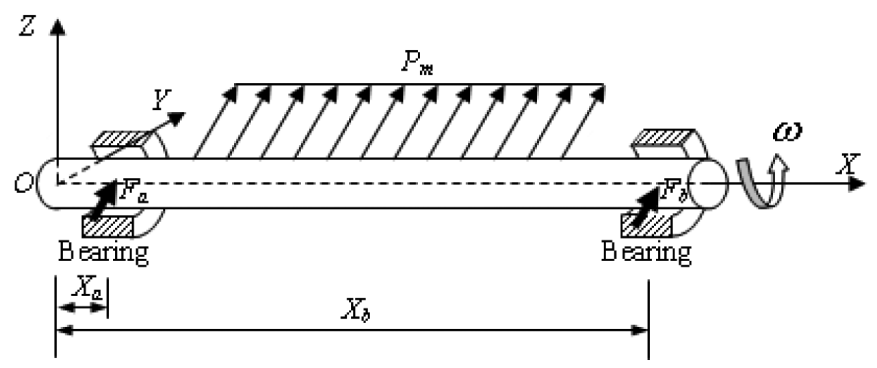

Fig. 1. A continuum rotor-bearing system excited by distributed electromagnetic load.

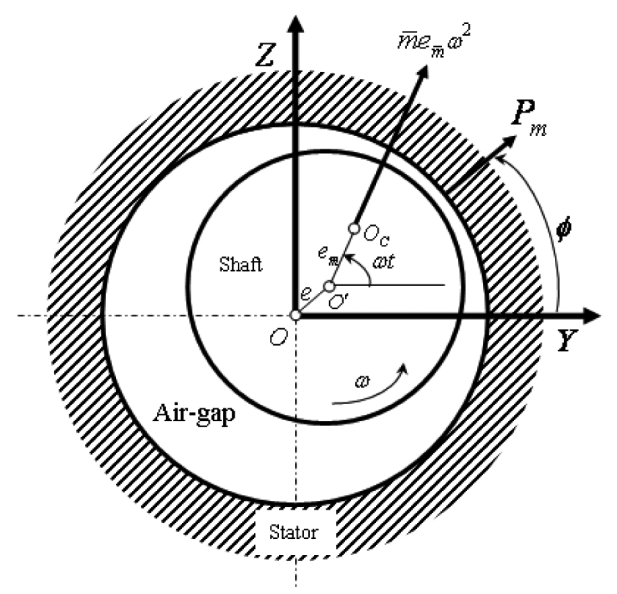

Fig. 2. The cross-section of the rotor shaft.

where $\bar{m}$ is mass density of the shaft per unit length and $\bar{m}=\rho_{s} \cdot \pi R^{2} ; \rho_{s}$ is density of the shaft; $R$ is radius of the shaft; $Y$ and $Z$ are function of $X$ and $t$, namely $Y(X, t)$ and $Z(X, t) ; E I$ is bending rigidity; $g$ is the gravitational acceleration; $\omega$ is rotation speed; $e_{\bar{m}}$ is mass eccentricity of the shaft's cross section; $\delta$ is the Dirac-delta function, $\left(F_{a Y}, F_{b Y}\right)$ and $\left(F_{a Z}, F_{b Z}\right)$ are components of the two oil-film forces in $Y$ - and $Z$-directions, respectively. The expression of the steady electromagnetic load, $P_{m}$ has been derived in [10], as

$$
P_{m}=\frac{\pi \gamma_{m} D e}{2 C_{e}}\left(1-\frac{e^{2}}{C_{e}^{2}}\right)^{-3 / 2}=\frac{\pi \gamma_{m} D\left(Y^{2}+Z^{2}\right)^{1 / 2}}{2 C_{e}}\left(1-\frac{Y^{2}+Z^{2}}{C_{e}^{2}}\right)^{-3 / 2}
$$

where $\gamma_{m}=B_{m}^{2} / \mu_{m}, B_{m}$ is magnetic flux density, $\mu_{m}$ is magnetic conductivity, $D$ is diameter of the shaft and $C_{e}$ is the mean length of air-gap.

The nonlinear oil-film forces can be expressed using the theoretical model of squeeze film damper bearing proposed in [26], as follows

$$
\left\{\begin{array}{l}
F_{Y}=-\mu_{o} \pi R L_{b}^{3}\left(\frac{\omega Z+2 \dot{Y}}{2\left(C^{2}-Y^{2}-Z^{2}\right)^{3 / 2}}+\frac{3 Y(Y \dot{Y}+Z \dot{Z})}{\left(C^{2}-Y^{2}-Z^{2}\right)^{5 / 2}}\right), \\
F_{Z}=-\mu_{o} \pi R L_{b}^{3}\left(\frac{2 \dot{Z}-\omega Y}{2\left(C^{2}-Y^{2}-Z^{2}\right)^{3 / 2}}+\frac{3 Z(Y \dot{Y}+Z \dot{Z})}{\left(C^{2}-Y^{2}-Z^{2}\right)^{5 / 2}}\right) .
\end{array}\right.
$$

where $\mu_{o}$ is viscosity of the oil film, $R$ is radius of the shaft, $L_{b}$ is length of the bearing and $C$ is the mean radial clearance of the bearing. Notice that $e^{2}=Y^{2}+Z^{2}, e \cos \phi=Y, e \sin \phi=Z$ and introduce non-dimensional quantities $\tau=\omega t, x=X / L, y=Y / C, z=Z / C, x_{a}=X_{a} / L$ and $x_{b}=X_{b} / L$. Substitution of Eqs (2) and (3) into Eq. (1) yields the equation of motion in the non-dimensional form 


$$
\left\{\begin{array}{l}
\ddot{y}+\kappa y^{\prime \prime \prime \prime}=G+\varepsilon_{\bar{m}} \cos \tau+\lambda_{m} y\left(1-C_{e}^{2}\left(y^{2}+z^{2}\right) / C^{2}\right)^{-3 / 2}-\left(\delta\left(x-x_{a}\right)+\delta\left(x-x_{b}\right)\right) \sigma_{f} f_{y}, \\
\ddot{z}+\kappa z^{\prime \prime \prime}=\varepsilon_{\bar{m}} \sin \tau+\lambda_{m} z\left(1-C_{e}^{2}\left(y^{2}+z^{2}\right) / C^{2}\right)^{-3 / 2}-\left(\delta\left(x-x_{a}\right)+\delta\left(x-x_{b}\right)\right) \sigma_{f} f_{z} .
\end{array}\right.
$$

where double dot and quadruple prime denote second-order and fourth-order derivatives of displacements with respect to $\tau$ and $x$, respectively, and

$$
\kappa=E I /\left(\bar{m} \omega^{2} L^{4}\right), G=g /\left(\omega^{2} C\right), \varepsilon_{\bar{m}}=e_{\bar{m}} / C, \lambda_{m}=\pi D \gamma_{m} /\left(2 \bar{m} \omega^{2} C_{e}\right), \sigma_{f}=\pi \mu_{o} R L_{b}^{3} /\left(\bar{m} \omega C^{3} L\right) .
$$

$f_{y}$ and $f_{z}$ are non-dimensional oil film forces expressed as

$$
\left\{\begin{array}{l}
f_{y}=\frac{z+2 \dot{y}}{2\left(1-y^{2}-z^{2}\right)^{3 / 2}}+\frac{3 y(y \dot{y}+z \dot{z})}{\left(1-y^{2}-z^{2}\right)^{5 / 2}}, \\
f_{z}=\frac{2 \dot{z}-y}{2\left(1-y^{2}-z^{2}\right)^{3 / 2}}+\frac{3 z(y \dot{y}+z \dot{z})}{\left(1-y^{2}-z^{2}\right)^{5 / 2}} .
\end{array}\right.
$$

Expanding the nonlinear forces of Eq. (5) into Taylor series in the vicinity of the equilibrium position of the shaft, the foregoing equation of motion can be rewritten as

$$
\left\{\begin{array}{l}
\ddot{y}+\kappa y^{\prime \prime \prime \prime}=G+\varepsilon_{\bar{m}} \cos \tau+\lambda_{m} y\left(1+\frac{3 C_{e}^{2}}{2 C^{2}} y^{2}+\frac{3 C_{e}^{2}}{2 C^{2}} z^{2}\right) \\
-\left(\delta\left(x-x_{a}\right)+\delta\left(x-x_{b}\right)\right) \sigma_{f}\left(\dot{y}+\frac{1}{2} z+\frac{9}{2} \dot{y} y^{2}+3 z \dot{z} y+\frac{3}{2} \dot{y} z^{2}+\frac{3}{4} z y^{2}+\frac{3}{4} z^{3}\right) \\
\ddot{z}+\kappa z^{\prime \prime \prime \prime}=\varepsilon_{\bar{m}} \sin \tau+\lambda_{m} z\left(1+\frac{3 C_{e}^{2}}{2 C^{2}} y^{2}+\frac{3 C_{e}^{2}}{2 C^{2}} z^{2}\right) \\
-\left(\delta\left(x-x_{a}\right)+\delta\left(x-x_{b}\right)\right) \sigma_{f}\left(\dot{z}-\frac{1}{2} y+\frac{3}{2} \dot{z} y^{2}+3 z \dot{y} y+\frac{9}{2} \dot{z} z^{2}-\frac{3}{4} y^{3}-\frac{3}{4} z^{2} y\right) .
\end{array}\right.
$$

The continuum rotor system can be reduced to a discretized one through approximating the transverse displacements into series forms:

$$
y(x, \tau)=\sum_{i=1}^{M} \xi_{i}(\tau) \phi_{i}(x), z(x, \tau)=\sum_{j=1}^{N} \eta_{j}(\tau) \phi_{j}(x)
$$

where $\phi_{k}(x)=\sin (k \pi x)$ is the $k$-th order trial function, $\xi_{i}(\tau)$ and $\eta_{j}(\tau)$ are $j$-th principal coordinates of the reduced system and $M$ is number of terms used in the series truncation. For low-frequency response we choose $M=2$. The governing equation of (6) becomes a truncated one as follows

$$
\left\{\begin{array}{l}
\ddot{\xi}_{1} \phi_{1}+\ddot{\xi}_{2} \phi_{2}+\kappa\left(\xi_{1} \phi_{1}^{\prime \prime \prime \prime}+\xi_{2} \phi_{2}^{\prime \prime \prime \prime}\right)=G+\varepsilon_{\bar{m}} \cos \tau \\
+\lambda_{m}\left(\left(\xi_{1} \phi_{1}+\xi_{2} \phi_{2}\right)+\frac{3 C_{e}^{2}}{2 C^{2}}\left(\xi_{1} \phi_{1}+\xi_{2} \phi_{2}\right)^{3}+\frac{3 C_{e}^{2}}{2 C^{2}}\left(\eta_{1} \phi_{1}+\eta_{2} \phi_{2}\right)^{2}\left(\xi_{1} \phi_{1}+\xi_{2} \phi_{2}\right)\right) \\
-\left(\delta\left(x-x_{a}\right)+\delta\left(x-x_{b}\right)\right) \sigma_{f}\left(\left(\dot{\xi}_{1} \phi_{1}+\dot{\xi}_{2} \phi_{2}\right)+\frac{1}{2}\left(\eta_{1} \phi_{1}+\eta_{2} \phi_{2}\right)\right. \\
+\frac{9}{2}\left(\dot{\xi}_{1} \phi_{1}+\dot{\xi}_{2} \phi_{2}\right)\left(\xi_{1} \phi_{1}+\xi_{2} \phi_{2}\right)^{2}+3\left(\eta_{1} \phi_{1}+\eta_{2} \phi_{2}\right)\left(\dot{\eta}_{1} \phi_{1}+\dot{\eta}_{2} \phi_{2}\right)\left(\xi_{1} \phi_{1}+\xi_{2} \phi_{2}\right) \\
\left.+\frac{3}{2}\left(\dot{\xi}_{1} \phi_{1}+\dot{\xi}_{2} \phi_{2}\right)\left(\eta_{1} \phi_{1}+\eta_{2} \phi_{2}\right)^{2}+\frac{3}{4}\left(\eta_{1} \phi_{1}+\eta_{2} \phi_{2}\right)\left(\xi_{1} \phi_{1}+\xi_{2} \phi_{2}\right)^{2}+\frac{3}{4}\left(\eta_{1} \phi_{1}+\eta_{2} \phi_{2}\right)^{3}\right) \\
\ddot{\eta}_{1} \phi_{1}+\ddot{\eta}_{2} \phi_{2}+\kappa\left(\eta_{1} \phi_{1}^{\prime \prime \prime \prime}+\eta_{2} \phi_{2}^{\prime \prime \prime \prime}\right)=\varepsilon_{\bar{m}} \sin \tau \\
+\lambda_{m}\left(\left(\eta_{1} \phi_{1}+\eta_{2} \phi_{2}\right)+\frac{3 C_{e}^{2}}{2 C^{2}}\left(\eta_{1} \phi_{1}+\eta_{2} \phi_{2}\right)\left(\xi_{1} \phi_{1}+\xi_{2} \phi_{2}\right)^{2}+\frac{3 C_{e}^{2}}{2 C^{2}}\left(\eta_{1} \phi_{1}+\eta_{2} \phi_{2}\right)^{3}\right) \\
-\left(\delta\left(x-x_{a}\right)+\delta\left(x-x_{b}\right)\right) \sigma_{f}\left(\left(\dot{\eta}_{1} \phi_{1}+\dot{\eta}_{2} \phi_{2}\right)-\frac{1}{2}\left(\xi_{1} \phi_{1}+\xi_{2} \phi_{2}\right)\right. \\
+\frac{3}{2}\left(\dot{\eta}_{1} \phi_{1}+\dot{\eta}_{2} \phi_{2}\right)\left(\xi_{1} \phi_{1}+\xi_{2} \phi_{2}\right)^{2}+3\left(\eta_{1} \phi_{1}+\eta_{2} \phi_{2}\right)\left(\dot{\xi}_{1} \phi_{1}+\dot{\xi}_{2} \phi_{2}\right)\left(\xi_{1} \phi_{1}+\xi_{2} \phi_{2}\right) \\
\left.+\frac{9}{2}\left(\dot{\eta}_{1} \phi_{1}+\dot{\eta}_{2} \phi_{2}\right)\left(\eta_{1} \phi_{1}+\eta_{2} \phi_{2}\right)^{2}-\frac{3}{4}\left(\xi_{1} \phi_{1}+\xi_{2} \phi_{2}\right)^{3}-\frac{3}{4}\left(\eta_{1} \phi_{1}+\eta_{2} \phi_{2}\right)^{2}\left(\xi_{1} \phi_{1}+\xi_{2} \phi_{2}\right)\right) .
\end{array}\right.
$$

Using the Galerkin's method, the foregoing can be further discretized as a group of ordinary differential equations 


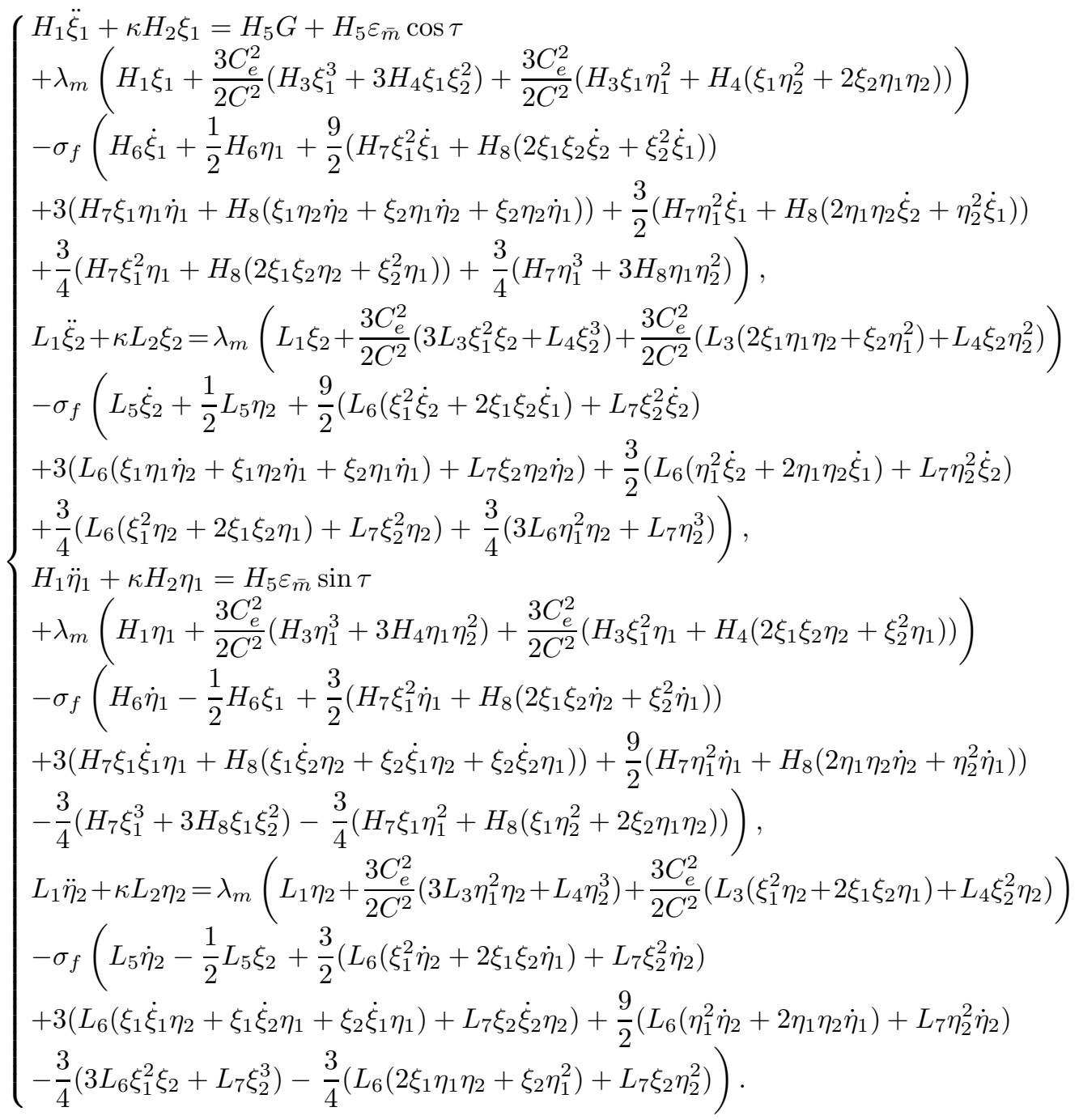

where coefficients $H_{1}, H_{2}, H_{3}, H_{4}, H_{5}, H_{6}, H_{7}, H_{8}, L_{1}, L_{2}, L_{3}, L_{4}, L_{5}, L_{6}$ and $L_{7}$ are integration constants expressed in Appendix A.

\section{Stability analyses for equilibrium position and primary resonance}

Equation (8) is linearized in the state space to analyze the stability of the rotor's equilibrium position. Let $\varepsilon_{\bar{m}}=$ 0 , one obtains

$$
\left(\begin{array}{c}
\dot{\xi}_{1} \\
\ddot{\xi}_{1} \\
\dot{\xi}_{2} \\
\ddot{\xi}_{2} \\
\dot{\eta}_{1} \\
\ddot{\eta}_{1} \\
\dot{\eta}_{2} \\
\ddot{\eta}_{2}
\end{array}\right)=\left(\begin{array}{cccccccc}
0 & 1 & 0 & 0 & 0 & 0 & 0 & 0 \\
-\frac{\kappa H_{2}}{H_{1}}+\lambda_{m} & -\frac{\sigma_{f} H_{6}}{H_{1}} & 0 & 0 & -\frac{\sigma_{f} H_{6}}{2 H_{1}} & 0 & 0 & 0 \\
0 & 0 & 0 & 1 & 0 & 0 & 0 & 0 \\
0 & 0 & -\frac{\kappa L_{2}}{L_{1}}+\lambda_{m} & -\frac{\sigma_{f} L_{5}}{L_{1}} & 0 & 0 & -\frac{\sigma_{f} L_{5}}{2 L_{1}} & 0 \\
0 & 0 & 0 & 0 & 0 & 1 & 0 & 0 \\
\frac{\sigma_{f} H_{6}}{2 H_{1}} & 0 & 0 & 0 & -\frac{\kappa H_{2}}{H_{1}}+\lambda_{m} & -\frac{\sigma_{f} H_{6}}{H_{1}} & 0 & 0 \\
0 & 0 & 0 & 0 & 0 & 0 & 0 & 1 \\
0 & 0 & \frac{\sigma_{f} L_{5}}{2 L_{1}} & 0 & 0 & 0 & -\frac{\kappa L_{2}}{L_{1}}+\lambda_{m} & -\frac{\sigma_{f} L_{5}}{L_{1}}
\end{array}\right)\left(\begin{array}{c}
\xi_{1} \\
\dot{\xi}_{1} \\
\xi_{2} \\
\dot{\xi}_{2} \\
\eta_{1} \\
\dot{\eta}_{1} \\
\eta_{2} \\
\dot{\eta}_{2}
\end{array}\right)
$$




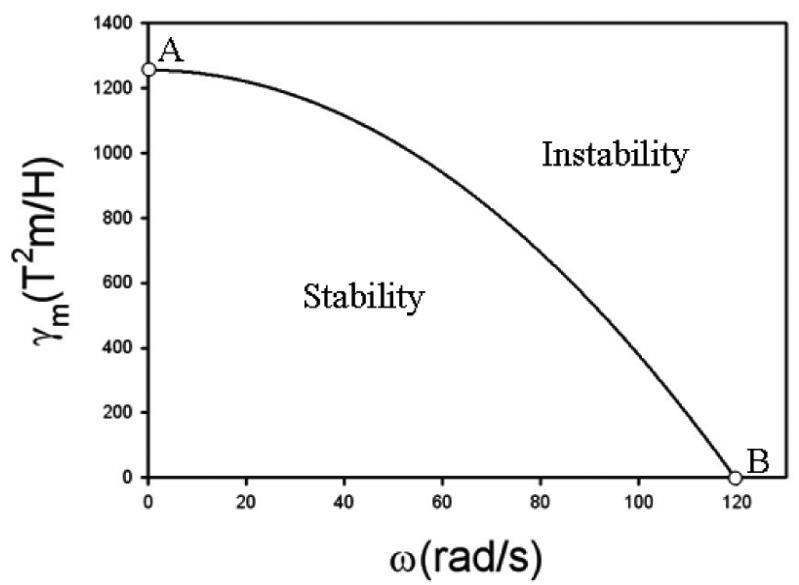

Fig. 3. The stability domain the parameter space of $\gamma_{m}$ and $\omega . D=0.2 \mathrm{~m}, \rho_{s}=7800 \mathrm{~kg} / \mathrm{m}^{3}, E=206 \mathrm{GPa}, C=0.0002 \mathrm{~m}, C_{e}=0.0002 \mathrm{~m}$, $L=3 \mathrm{~m}, L_{b}=0.05 \mathrm{~m}, x_{a}=0.08, x_{b}=0.92, \mu_{o}=0.018 \mathrm{~Pa} \cdot \mathrm{s}$.

The stability of the rotor's equilibrium can be obtained in parameter space using the Routh-Hurwitz criterion. To present it, the control parameters are chosen to be rotation speed $\omega$ and electromagnetic parameter $\gamma_{m}$. Let $D=$ $0.2 \mathrm{~m}, \rho_{s}=7800 \mathrm{~kg} / \mathrm{m}^{3}, E=206 \mathrm{GPa}, C=0.0002 \mathrm{~m}, C_{e}=0.0002 \mathrm{~m}, L=3 \mathrm{~m}, L_{b}=0.05 \mathrm{~m}, x_{a}=0.08, x_{b}=$ 0.92 and $\mu_{o}=0.018 \mathrm{~Pa} \cdot \mathrm{s}$. The stability of the equilibrium is depicted in Fig. 3, where curve AB separates the domain of asymptotic stability from that of instability. Notice that at least one pair of complex conjugated eigenvalues is zero for all points on curve $\mathrm{AB}$ corresponding to the Andronov-Hopf bifurcation. The equilibrium position of the rotor will be lost through the Andronov-Hopf bifurcation by increasing $\omega$ and/or increasing $\gamma_{m}$. Afterwards, periodic motion of the rotor will appear.

For primary resonance of the periodic motion we choose $M=1$ and rewrite Eq. (8) as

$$
\left\{\begin{array}{l}
\ddot{\xi}_{1}+\frac{\omega_{0}^{2}}{\omega^{2}} \xi_{1}=\frac{H_{5}}{H_{1}} G+\frac{H_{5}}{H_{1}} \varepsilon_{\bar{m}} \cos \tau+\frac{\lambda_{m}}{H_{1}}\left(\frac{3 C_{e}^{2}}{2 C^{2}} H_{3} \xi_{1}^{3}+\frac{3 C_{e}^{2}}{2 C^{2}} H_{3} \xi_{1} \eta_{1}^{2}\right) \\
-\frac{\sigma_{f}}{H_{1}}\left(H_{6} \dot{\xi}_{1}+\frac{1}{2} H_{6} \eta_{1}+\frac{9}{2} H_{7} \xi_{1}^{2} \dot{\xi}_{1}+3 H_{7} \xi_{1} \eta_{1} \dot{\eta}_{1}+\frac{3}{2} H_{7} \eta_{1}^{2} \dot{\xi}_{1}+\frac{3}{4} H_{7} \xi_{1}^{2} \eta_{1}+\frac{3}{4} H_{7} \eta_{1}^{3}\right), \\
\ddot{\eta}_{1}+\frac{\omega_{0}^{2}}{\omega^{2}} \eta_{1}=\frac{H_{5}}{H_{1}} \varepsilon_{\bar{m}} \sin \tau+\frac{\lambda_{m}}{H_{1}}\left(\frac{3 C_{e}^{2}}{2 C^{2}} H_{3} \eta_{1}^{3}+\frac{3 C_{e}^{2}}{2 C^{2}} H_{3} \xi_{1}^{2} \eta_{1}\right) \\
-\frac{\sigma_{f}}{H_{1}}\left(H_{6} \dot{\eta}_{1}-\frac{1}{2} H_{6} \xi_{1}+\frac{3}{2} H_{7} \xi_{1}^{2} \dot{\eta}_{1}+3 H_{7} \xi_{1} \dot{\xi}_{1} \eta_{1}+\frac{9}{2} H_{7} \eta_{1}^{2} \dot{\eta}_{1}-\frac{3}{4} H_{7} \xi_{1}^{3}-\frac{3}{4} H_{7} \xi_{1} \eta_{1}^{2}\right) .
\end{array}\right.
$$

where $\omega_{0}=\omega \sqrt{\kappa H_{3} / H_{1}-\lambda_{m}}$ is the natural frequency of the linear system. Assume that $\omega_{0}^{2}=(1-\varepsilon \sigma) \omega^{2}$. Equation (10) can be rewritten as

$$
\left\{\begin{array}{l}
\ddot{\xi}_{1}+\xi_{1}=\frac{H_{5}}{H_{1}} G+\varepsilon\left(\sigma \xi_{1}+\frac{H_{5}}{H_{1}} \varepsilon_{\bar{m}} \cos \tau+\frac{\lambda_{m}}{H_{1}}\left(\frac{3 C_{e}^{2}}{2 C^{2}} H_{3} \xi_{1}^{3}+\frac{3 C_{e}^{2}}{2 C^{2}} H_{3} \xi_{1} \eta_{1}^{2}\right)\right. \\
\left.-\frac{\sigma_{f}}{H_{1}}\left(H_{6} \dot{\xi}_{1}+\frac{1}{2} H_{6} \eta_{1}+\frac{9}{2} H_{7} \xi_{1}^{2} \dot{\xi}_{1}+3 H_{7} \xi_{1} \eta_{1} \dot{\eta}_{1}+\frac{3}{2} H_{7} \eta_{1}^{2} \dot{\xi}_{1}+\frac{3}{4} H_{7} \xi_{1}^{2} \eta_{1}+\frac{3}{4} H_{7} \eta_{1}^{3}\right)\right), \\
\ddot{\eta}_{1}+\eta_{1}=\varepsilon\left(\sigma \eta_{1}+\frac{H_{5}}{H_{1}} \varepsilon_{\bar{m}} \sin \tau+\frac{\lambda_{m}}{H_{1}}\left(\frac{3 C_{e}^{2}}{2 C^{2}} H_{3} \eta_{1}^{3}+\frac{3 C_{e}^{2}}{2 C^{2}} H_{3} \xi_{1}^{2} \eta_{1}\right)\right. \\
\left.-\frac{\sigma_{f}}{H_{1}}\left(H_{6} \dot{\eta}_{1}-\frac{1}{2} H_{6} \xi_{1}+\frac{3}{2} H_{7} \xi_{1}^{2} \dot{\eta}_{1}+3 H_{7} \xi_{1} \dot{\xi}_{1} \eta_{1}+\frac{9}{2} H_{7} \eta_{1}^{2} \dot{\eta}_{1}-\frac{3}{4} H_{7} \xi_{1}^{3}-\frac{3}{4} H_{7} \xi_{1} \eta_{1}^{2}\right)\right) .
\end{array}\right.
$$

where $\varepsilon \ll 1$ is the small parameter of the perturbed system, $\sigma$ is the tuning parameter. Equation (11) can be expressed in a shortened form

$$
\left\{\begin{array}{l}
\ddot{\xi}_{1}+\xi_{1}=f_{g}+\varepsilon F_{1}\left(\xi_{1}, \eta_{1}, \dot{\xi}_{1}, \dot{\eta}_{1}, \tau\right), \\
\ddot{\eta}_{1}+\eta_{1}=\varepsilon F_{2}\left(\xi_{1}, \eta_{1}, \dot{\xi}_{1}, \dot{\eta}_{1}, \tau\right) .
\end{array}\right.
$$


where $f_{g}=G H_{5} / H_{1}$, and

$$
\left\{\begin{array}{l}
F_{1}\left(\xi_{1}, \eta_{1}, \dot{\xi}_{1}, \dot{\eta}_{1}, \tau\right)=\sigma \xi_{1}+\frac{H_{5}}{H_{1}} \varepsilon_{\bar{m}} \cos \tau+\frac{\lambda_{m}}{H_{1}}\left(\frac{3 C_{e}^{2}}{2 C^{2}} H_{3} \xi_{1}^{3}+\frac{3 C_{e}^{2}}{2 C^{2}} H_{3} \xi_{1} \eta_{1}^{2}\right) \\
-\frac{\sigma_{f}}{H_{1}}\left(H_{6} \dot{\xi}_{1}+\frac{1}{2} H_{6} \eta_{1}+\frac{9}{2} H_{7} \xi_{1}^{2} \dot{\xi}_{1}+3 H_{7} \xi_{1} \eta_{1} \dot{\eta}_{1}+\frac{3}{2} H_{7} \eta_{1}^{2} \dot{\xi}_{1}+\frac{3}{4} H_{7} \xi_{1}^{2} \eta_{1}+\frac{3}{4} H_{7} \eta_{1}^{3}\right), \\
F_{2}\left(\xi_{1}, \eta_{1}, \dot{\xi}_{1}, \dot{\eta}_{1}, \tau\right)=\sigma \eta_{1}+\frac{H_{5}}{H_{1}} \varepsilon_{\bar{m}} \sin \tau+\frac{\lambda_{m}}{H_{1}}\left(\frac{3 C_{e}^{2}}{2 C^{2}} H_{3} \eta_{1}^{3}+\frac{3 C_{e}^{2}}{2 C^{2}} H_{3} \xi_{1}^{2} \eta_{1}\right) \\
-\frac{\sigma_{f}}{H_{1}}\left(H_{6} \dot{\eta}_{1}-\frac{1}{2} H_{6} \xi_{1}+\frac{3}{2} H_{7} \xi_{1}^{2} \dot{\eta}_{1}+3 H_{7} \xi_{1} \dot{\xi}_{1} \eta_{1}+\frac{9}{2} H_{7} \eta_{1}^{2} \dot{\eta}_{1}-\frac{3}{4} H_{7} \xi_{1}^{3}-\frac{3}{4} H_{7} \xi_{1} \eta_{1}^{2}\right) .
\end{array}\right.
$$

Assume $\varphi=\tau+\theta, \xi_{1}=a \cos \varphi+f_{g}, \eta_{1}=a \sin \varphi$, where $a$ and $\theta$ are slowly varying functions of time determined through

$$
\left\{\begin{array}{l}
\dot{a}=\varepsilon\left(-F_{1} \sin \varphi+F_{2} \cos \varphi\right), \\
a \dot{\theta}=\varepsilon\left(F_{1} \cos \varphi+F_{2} \sin \varphi\right) .
\end{array}\right.
$$

Introducing the Krylov-Bogliubov transformation: $\dot{y}=\varepsilon Y_{1}$ and $\dot{\theta}=\varepsilon Z_{1}$, and using the averaging form of Eq. (14), one obtains

$$
\left(\begin{array}{c}
Y_{1} \\
a Z_{1}
\end{array}\right)=\frac{1}{2 \pi} \int_{0}^{2 \pi}\left(\begin{array}{c}
-F_{1} \sin \varphi+F_{2} \cos \varphi \\
F_{1} \cos \varphi+F_{2} \sin \varphi
\end{array}\right) d \varphi
$$

Substitution of Eq. (13) into Eq. (15) yields the steady state response

$$
\left\{\begin{array}{l}
Y_{1}=-\left(\frac{\sigma_{f} H_{6}}{2 H_{1}}+\frac{3 \sigma_{f} H_{7} f_{g}^{2}}{2 H_{1}}\right) a-\frac{3 \sigma_{f} H_{7}}{4 H_{1}} a^{3}-\frac{H_{5} \varepsilon_{\bar{m}}}{H_{1}} \sin \theta, \\
Z_{1}=\sigma+\frac{3 \lambda_{m} C_{e}^{2} H_{3} f_{g}^{2}}{C^{2} H_{1}}+\frac{3 \lambda_{m} C_{e}^{2} H_{3}}{2 C^{2} H_{1}} a^{2}+\frac{H_{5} \varepsilon_{\bar{m}}}{H_{1} a} \cos \theta .
\end{array}\right.
$$

Eliminating $\theta$, one obtains the amplitude-frequency relation of primary resonance

$$
\sigma_{f}^{2}\left(\frac{H_{6}}{2 H_{1}}+\frac{3 H_{7} f_{g}^{2}}{2 H_{1}}+\frac{3 H_{19} a^{2}}{4 H_{1}}\right)^{2}+\left(\sigma+\frac{3 \lambda_{m} C_{e}^{2} H_{3} f_{g}^{2}}{C^{2} H_{1}}+\frac{3 \lambda_{m} C_{e}^{2} H_{3} a^{2}}{2 C^{2} H_{1}}\right)^{2}=\left(\frac{\varepsilon_{\bar{m}} H_{5}}{a H_{1}}\right)^{2}
$$

as plotted in Fig. 4, where the parameters are $D=0.2 \mathrm{~m}, \omega=200 \mathrm{rad} / \mathrm{s}$ and $\gamma_{m}=1000 \mathrm{~T}^{2} \mathrm{~m} / \mathrm{H}$. The amplitudefrequency curve shows the characteristic of a soft spring system due to nonlinear electromagnetic stiffness. Further, it can be seen that amplitude $a$ jumps as $\sigma$ takes critical values on the curve, and the number of steady response of $a$ changes accordingly.

\section{Numerical simulation and discussion}

In this section the fourth-order Runge-Kutta method is used to solve the nonlinear system of Eq. (8) for transient transverse vibration of the rotor. Let $\rho_{s}=7800 \mathrm{~kg} / \mathrm{m}^{3}, E=206 \mathrm{GPa}, L=3 \mathrm{~m}, x_{a}=0.08, x_{b}=0.92, C=$ $0.001 D, L_{b}=D / 4, e_{\bar{m}}=0.0001 \mathrm{~m}$. The $y$ - and $z$-displacements of the mid-span point will be illustrated.

\subsection{Influence of rotation speed}

Let $D=0.25 \mathrm{~m}, \gamma_{m}=1000 \mathrm{~T}^{2} \mathrm{~m} / \mathrm{H}, \mu_{o}=0.001 \mathrm{~Pa} \cdot \mathrm{s}, C_{e}=D / 1600$. Figure 5 shows the bifurcation diagram of $y$-displacement of the mid-span point excited by electromagnetic load versus the rotation speed. Figure 6 is the bifurcation diagram of the same displacement excited by oil-film forces versus the rotation speed. The two figures show that the contributions of electromagnetic load and oil-film force to the displacement response are very different. Figure 7 is the bifurcation diagram of $y$-displacement of the same point excited by both of the electromagnetic load and the oil-film force. It is noticed that the bifurcation rotation speeds for appearance of quasi-periodic motion are 


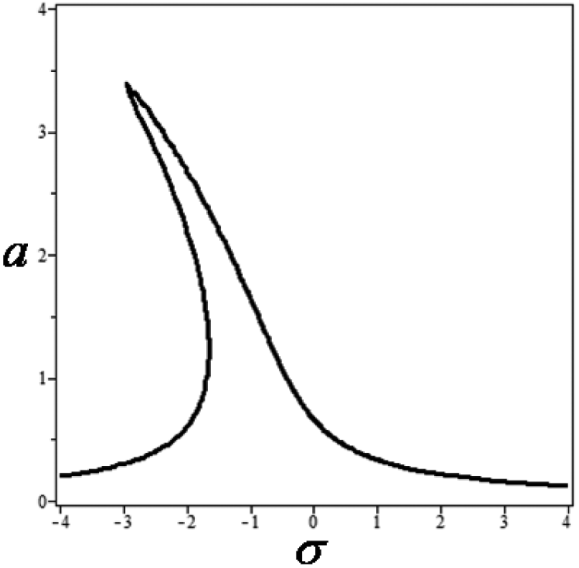

Fig. 4. The amplitude-frequency response curve near primary resonance. $D=0.2 \mathrm{~m}, \omega=200 \mathrm{rad} / \mathrm{s}, \gamma_{m}=1000 \mathrm{~T}^{2} \mathrm{~m} / \mathrm{H}, \rho_{s}=$ $7800 \mathrm{~kg} / \mathrm{m}^{3}, E=206 \mathrm{GPa}, C=0.0002 \mathrm{~m}, C_{e}=0.0002 \mathrm{~m}, L=$ $3 \mathrm{~m}, L_{b}=0.05 \mathrm{~m}, e_{\bar{m}}=0.0001 \mathrm{~m}, x_{a}=0.08, x_{b}=0.92, \mu_{o}=$ $0.018 \mathrm{~Pa} \cdot \mathrm{s}$

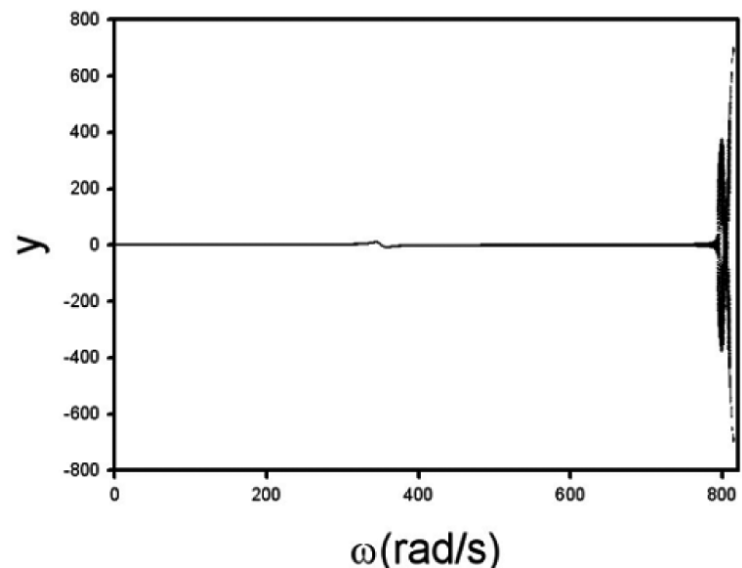

Fig. 6. The bifurcation diagram of $y$-displacement at the mid-span point versus rotation speed excited by oil-film force. $D=0.25 \mathrm{~m}$, $\gamma_{m}=1000 \mathrm{~T}^{2} \mathrm{~m} / \mathrm{H}, \rho_{s}=7800 \mathrm{~kg} / \mathrm{m}^{3}, E=206 \mathrm{GPa}, C=$ $0.001 D, C_{e}=D / 1600, L=3 \mathrm{~m}, L_{b}=D / 4, e_{\bar{m}}=0.0001 \mathrm{~m}$, $x_{a}=0.08, x_{b}=0.92, \mu_{o}=0.001 \mathrm{~Pa} \cdot \mathrm{s}$.

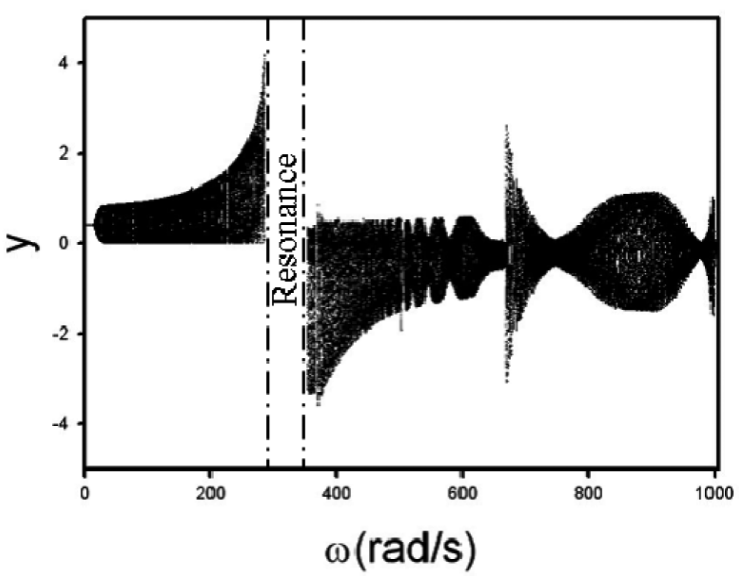

Fig. 5. The bifurcation diagram of $y$-displacement at the mid-span point versus rotation speed excited by electromagnetic load. $D=$ $0.25 \mathrm{~m}, \gamma_{m}=1000 \mathrm{~T}^{2} \mathrm{~m} / \mathrm{H}, \rho_{s}=7800 \mathrm{~kg} / \mathrm{m}^{3}, E=206 \mathrm{GPa}, C=$ $0.001 D, C_{e}=D / 1600, L=3 \mathrm{~m}, L_{b}=D / 4, e_{\bar{m}}=0.0001 \mathrm{~m}$, $x_{a}=0.08, x_{b}=0.92, \mu_{o}=0.001 \mathrm{~Pa} \cdot \mathrm{s}$.

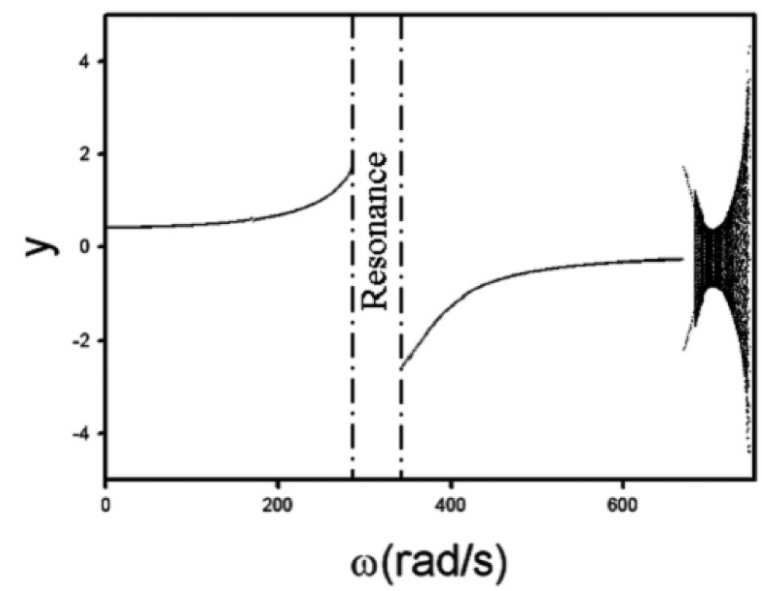

Fig. 7. The bifurcation diagram of $y$-displacement at the mid-span point versus rotation speed excited by both of electromagnetic load and oil-film force. $D=0.25 \mathrm{~m}, \gamma_{m}=1000 \mathrm{~T}^{2} \mathrm{~m} / \mathrm{H}, \rho_{s}=$ $7800 \mathrm{~kg} / \mathrm{m}^{3}, E=206 \mathrm{GPa}, C=0.001 D, C_{e}=D / 1600, L=$ $3 \mathrm{~m}, L_{b}=D / 4, e_{\bar{m}}=0.0001 \mathrm{~m}, x_{a}=0.08, x_{b}=0.92, \mu_{o}=$ $0.001 \mathrm{~Pa} \cdot \mathrm{s}$.

$\omega=14 \mathrm{rad} / \mathrm{s}$ in Fig. 5, $\omega=478 \mathrm{rad} / \mathrm{s}$ in Fig. 6 and $\omega=684 \mathrm{rad} / \mathrm{s}$ in Fig. 7, respectively. This shows that the oil-film force tends to maintain periodic motion at relatively low rotation speeds and period-2 motion can be found before quasi-periodic motion appears. The resonance amplitude of the rotor is seen reduced as well due to the damping of bearings. The electromagnetic load, by contrast, tends to make quasi-periodic motion appear at relatively high rotation speed and increase the amplitude at the resonance. Eventually, the displacement amplitude grows drastically with very high rotation speed as shown in Figs 6 and 7, and the motion diverges due to the unstable motion of the oil-film. Further, the displacement amplitude is much smaller in Fig. 7 than in Fig. 6 when divergence happens.

Let $D=0.25 \mathrm{~m}$. Figures 8 (a) and 8(b) are bifurcation diagrams of $y$ - and $z$-displacements of the mid-span point of shaft. It can be seen that the motion is synchronous and period- 1 for $\omega$ less than $286 \mathrm{rad} / \mathrm{s}$. As the speed increases, primary resonance appears and the amplitudes of displacements approach infinity. For $345 \mathrm{rad} / \mathrm{s}<\omega<670 \mathrm{rad} / \mathrm{s}$ the motion resumes period- 1 synchronous. Then, oil whirling begins at $\omega=670 \mathrm{rad} / \mathrm{s}$ and the motion is period- 2 up 


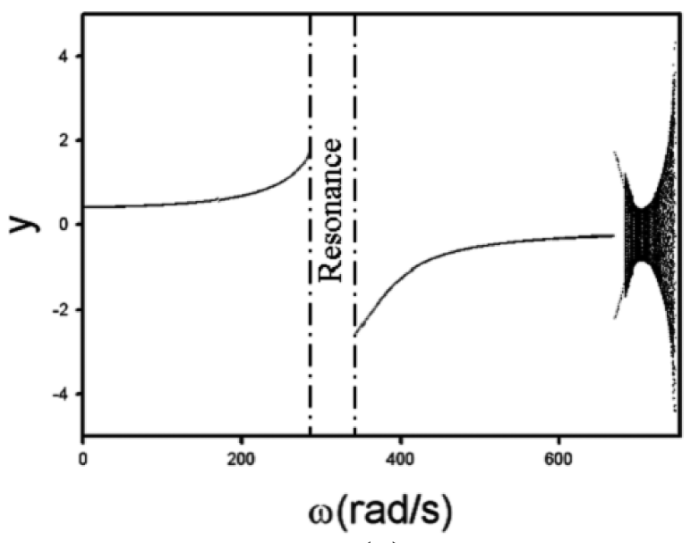

(a)

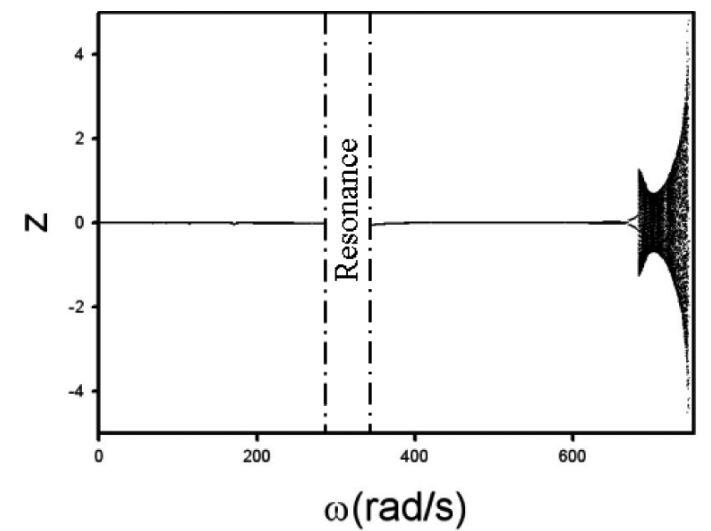

(b)

Fig. 8. The bifurcation diagram of displacements at the mid-span point versus rotation speed. (a) $y$-displacement; (b) $z$-displacement. $D=$ $0.25 \mathrm{~m}, \gamma_{m}=1000 \mathrm{~T}^{2} \mathrm{~m} / \mathrm{H}, \rho_{s}=7800 \mathrm{~kg} / \mathrm{m}^{3}, E=206 \mathrm{GPa}, C=0.001 D, C_{e}=D / 16000, L=3 \mathrm{~m}, L_{b}=D / 4, e_{\bar{m}}=0.0001 \mathrm{~m}, x_{a}=$ $0.08, x_{b}=0.92, \mu_{o}=0.001 \mathrm{~Pa} \cdot \mathrm{s}$.

to $\omega=683 \mathrm{rad} / \mathrm{s}$. The motion becomes quasi-periodic in the range of $683 \mathrm{rad} / \mathrm{s}<\omega<745 \mathrm{rad} / \mathrm{s}$ as the rotation speed increases.

In the following, diagrams of time history, shaft orbital trajectory, Poincaré section and the fast Fourier transformation (FFT) of $y$-displacement at the mid-span point of the rotor are presented for further understanding the vibration response of the system. Figures 9 (a) through 9 (d) confirm that the motion is period-1 synchronous with a frequency of $200 \mathrm{rad} / \mathrm{s}$. Figures 10(a) through 10(d) show the same characteristics of the motion with $\omega=350 \mathrm{rad} / \mathrm{s}$ but the displacement amplitude is larger. For $\omega=680 \mathrm{rad} / \mathrm{s}$ the motion is identified as period-2 based on Figs 11(a) through $11(\mathrm{~d})$ as an one-half frequency component appears, which indicates the beginning of oil whirling. As the speed increases, the motion becomes quasi-periodic as shown in Figs 12(a) through 12(d) when $\omega=700 \mathrm{rad} / \mathrm{s}$. There are two components of incommensurate frequencies in the motion response based on Fig. 12(d), and the Poincaré section in Fig. 12(c) forms a ring of densely spotted phase points. For $\omega=740 \mathrm{rad} / \mathrm{s}$ the motion is still quasi-periodic as seen in Figs 13(a) through 13(d), but the amplitude of the subharmonic frequency enlarges with increasing speed, which indicates the occurring of oil whip.

\subsection{Influence of electromagnetic parameter}

Electromagnetic parameter is another issue that influences dynamic characteristics of the rotor system. Assume $D=0.25 \mathrm{~m}, \omega=600 \mathrm{rad} / \mathrm{s}, \mu_{0}=0.001 \mathrm{~Pa} \cdot \mathrm{s}, C_{e}=D / 1600$. Figures 14 (a) and 14 (b) present the bifurcation diagrams of $y$ - and $z$-displacements of the mid-span point versus $\gamma_{m}$. It is found out that the quasi-periodic motion occurring when $0<\gamma_{m}<1058 \mathrm{~T}^{2} \mathrm{~m} / \mathrm{H}$ and the rotor is in motion of period-1 with the same frequency as the rotation speed for $1058<\gamma_{m}<2722 \mathrm{~T}^{2} \mathrm{~m} / \mathrm{H}$. Oil whirling begins when $\gamma_{m}=2722 \mathrm{~T}^{2} \mathrm{~m} / \mathrm{H}$ and the motion becomes period-2 through a period-doubling bifurcation. The amplitudes of the two displacements both jump at $\gamma_{m}=4210 \mathrm{~T}^{2} \mathrm{~m} / \mathrm{H}$. For $\gamma_{m}>4210 \mathrm{~T}^{2} \mathrm{~m} / \mathrm{H}$ the period-2 motion becomes unstable and is replaced by quasi-periodic motion. The motion remains quasi-periodic and gradually decreases with larger $\gamma_{m}$, nevertheless, period-2 motion still can be found in two little region.

More details regarding the quasi-periodic motion in the $y$-direction with $\gamma_{m}=100 \mathrm{~T}^{2} \mathrm{~m} / \mathrm{H}$ are shown in Figs 15 (a) through 15(d). Then, the motion becomes period-1 motion for $\gamma_{m}=2000 \mathrm{~T}^{2} \mathrm{~m} / \mathrm{H}$ as shown in Figs 16(a) through 16(d). The motion of period-2 appears and oil whirling happens when $\gamma_{m}=4000 \mathrm{~T}^{2} \mathrm{~m} / \mathrm{H}$ as shown in Figs 17 (a) through 17(d). Notice that the one-half frequency has dominating amplitude over the frequency of rotation. For $\gamma_{m}=4500 \mathrm{~T}^{2} \mathrm{~m} / \mathrm{H}$ the dynamic characteristics of the motion is presented in Figs 18(a) through 18(d). The motion is found to be quasi-periodic and has four frequencies in Fig. 18(d). The appearance of beat can be found in Fig. 18(a), because there are three adjacent frequencies in Fig. 18(d). 


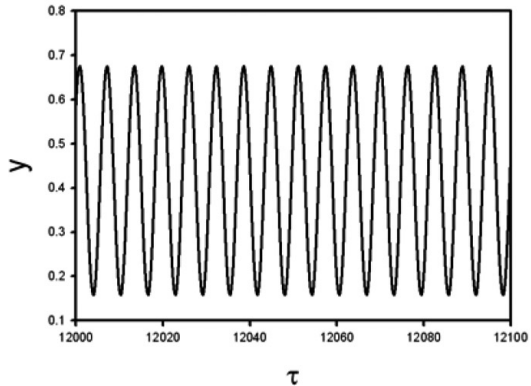

(a)

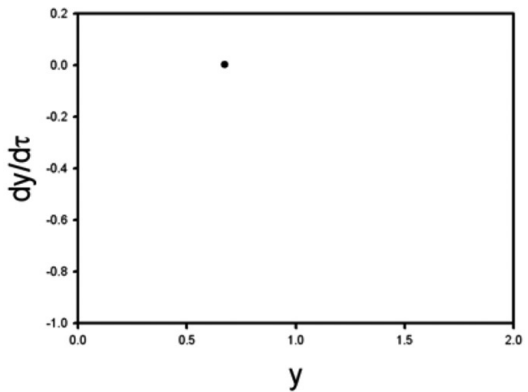

(c)

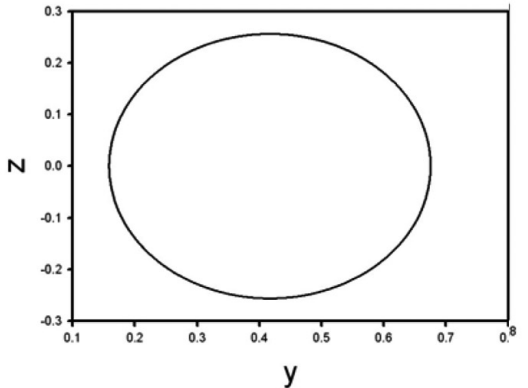

(b)

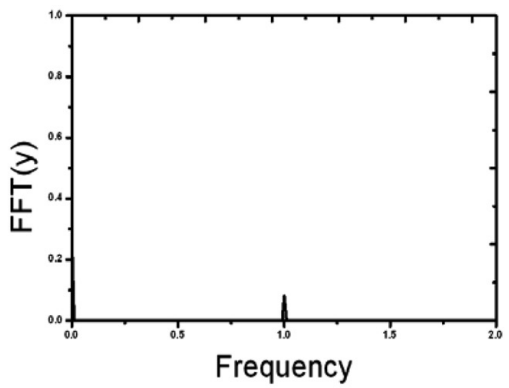

(d)

Fig. 9. Dynamic characteristic of the y-displacement of the mid-span point with $\omega=200 \mathrm{rad} / \mathrm{s}$. (a) Time history; (b) Orbital trajectory; (c) Poincaré section; (d) FFT.

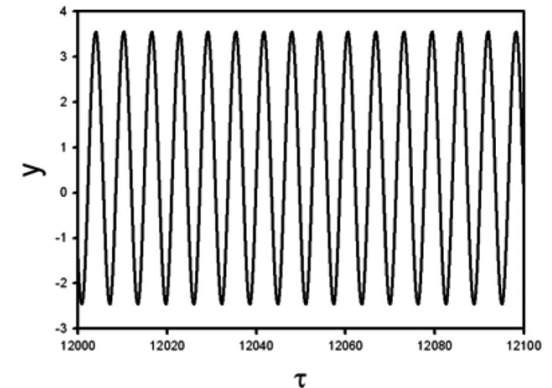

(a)

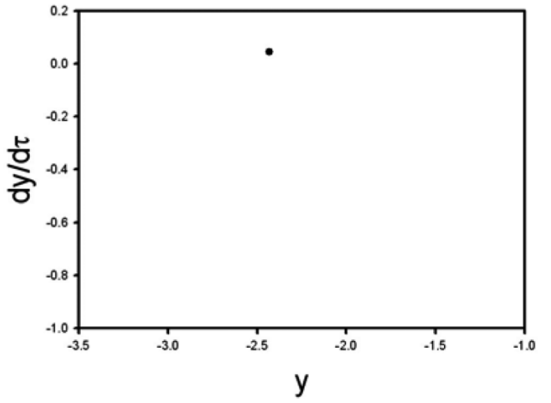

(c)

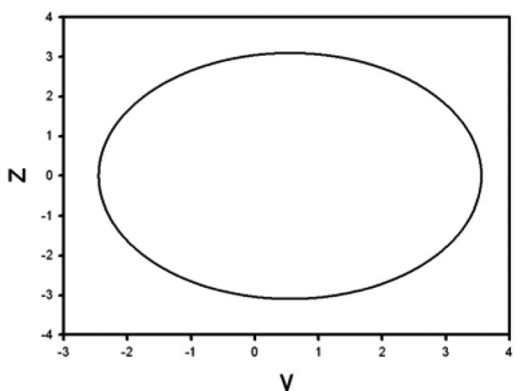

(b)

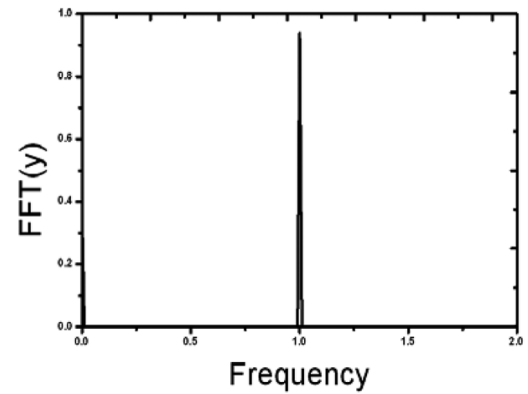

(d)

Fig. 10. Dynamic characteristic of the y-displacement of the mid-span point with $\omega=350 \mathrm{rad} / \mathrm{s}$. (a) Time history; (b) Orbital trajectory; (c) Poincaré section; (d) FFT. 


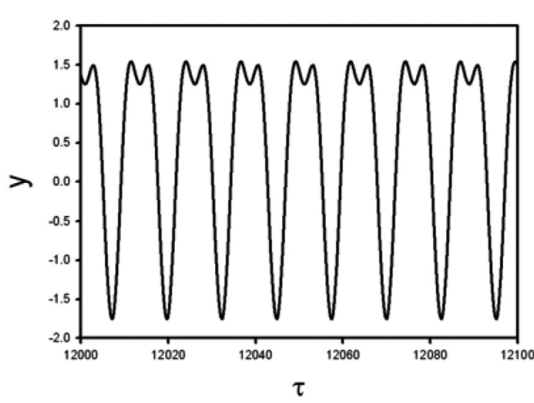

(a)

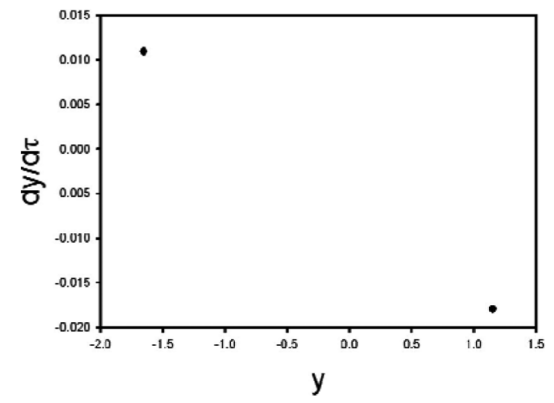

(c)

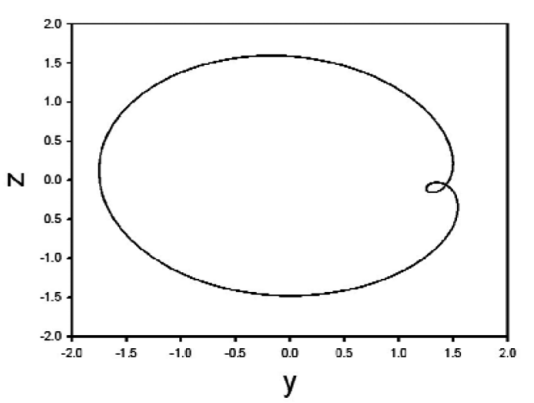

(b)

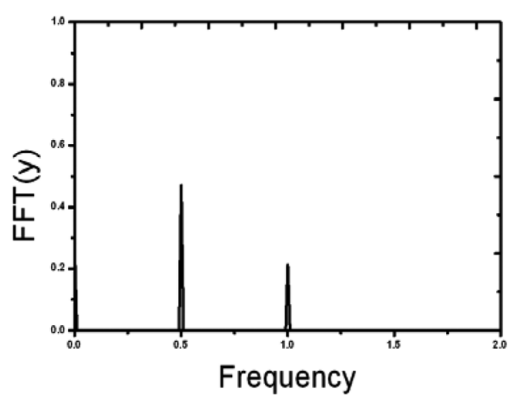

(d)

Fig. 11. Dynamic characteristic of the y-displacement of the mid-span point with $\omega=680 \mathrm{rad} / \mathrm{s}$. (a) Time history; (b) Orbital trajectory; (c) Poincaré section; (d) FFT.

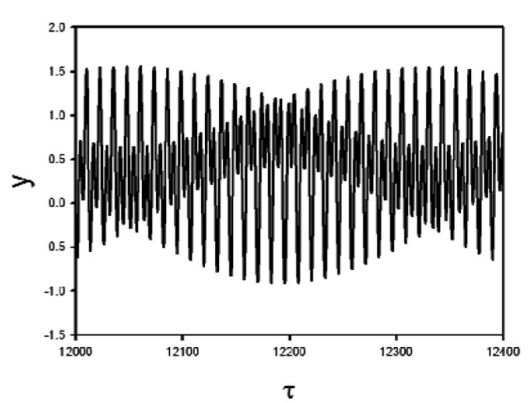

(a)

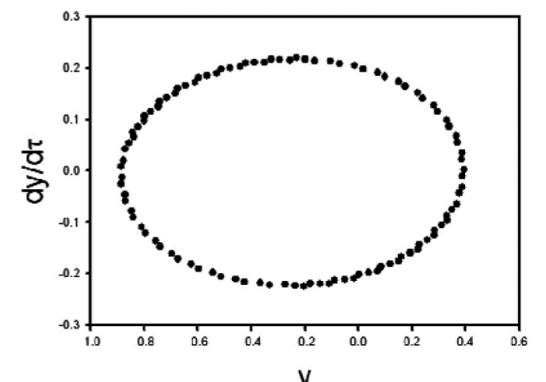

(c)

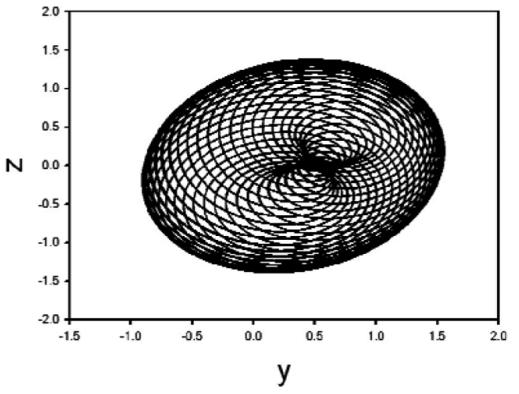

(b)

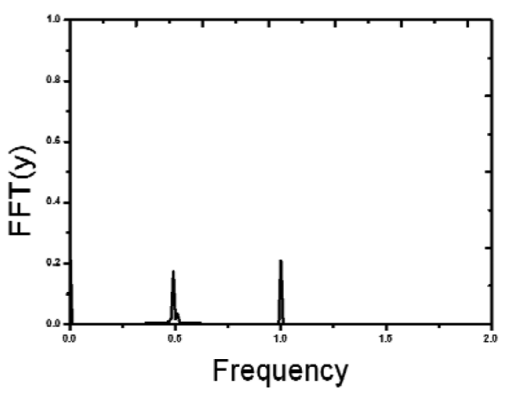

(d)

Fig. 12. Dynamic characteristic of the y-displacement of the mid-span point with $\omega=700 \mathrm{rad} / \mathrm{s}$. (a) Time history; (b) Orbital trajectory; (c) Poincaré section; (d) FFT. 


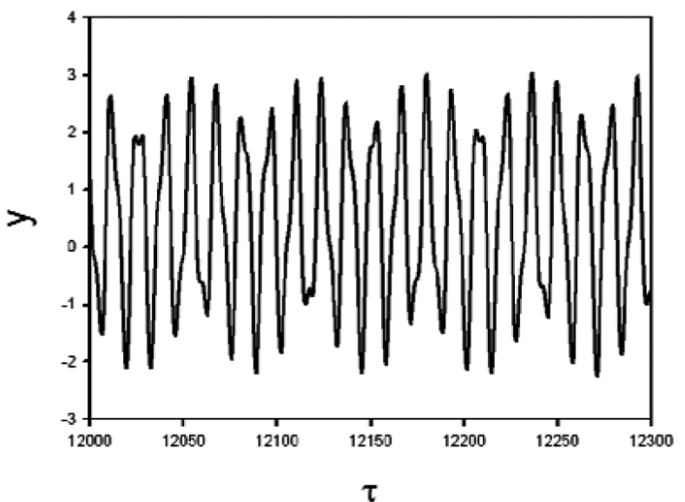

(a)

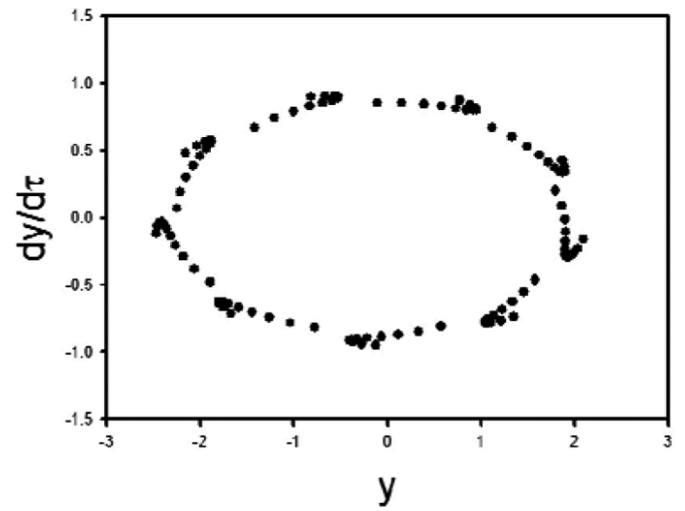

(c)

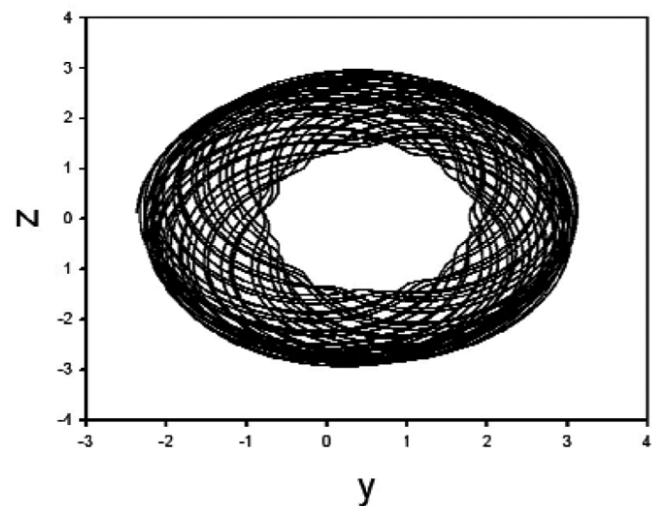

(b)

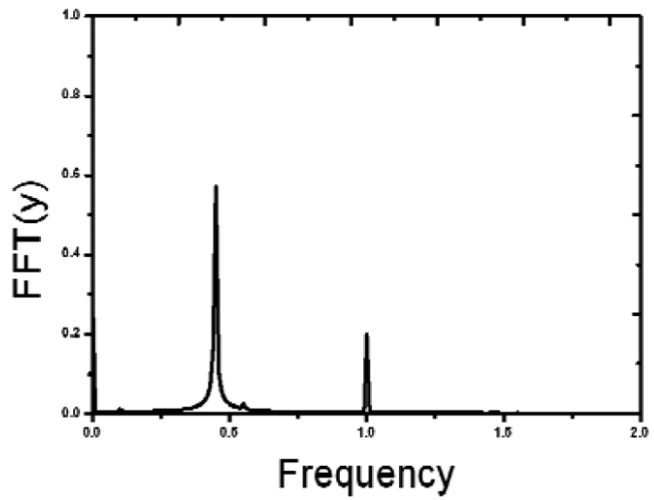

(d)

Fig. 13. Dynamic characteristic of the y-displacement of the mid-span point with $\omega=740 \mathrm{rad} / \mathrm{s}$. (a) Time history; (b) Orbital trajectory; (c) Poincaré section; (d) FFT.

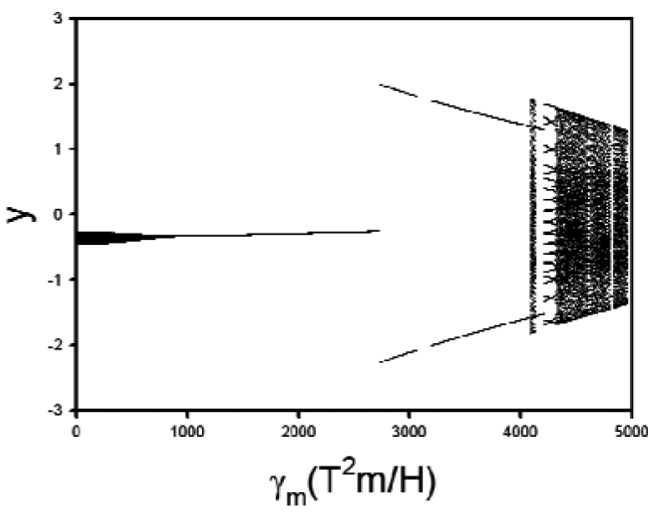

(a)

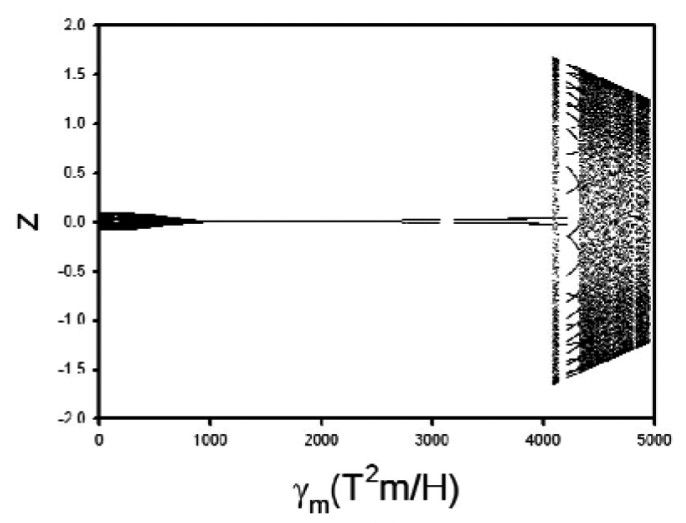

(b)

Fig. 14. Bifurcation diagram of displacement versus electromagnetic parameter $\gamma_{m}$. (a) $y$-displacement; (b) $z$-displacement. $D=0.25 \mathrm{~m}$, $\omega=$ $600 \mathrm{rad} / \mathrm{s}, \rho_{s}=7800 \mathrm{~kg} / \mathrm{m}^{3}, E=206 \mathrm{GPa}, C=0.001 D, C_{e}=D / 1600, L=3 \mathrm{~m}, L_{b}=D / 4, e_{\bar{m}}=0.0001 \mathrm{~m}, x_{a}=0.08, x_{b}=0.92$, $\mu_{o}=0.001 \mathrm{~Pa} \cdot \mathrm{s}$ 


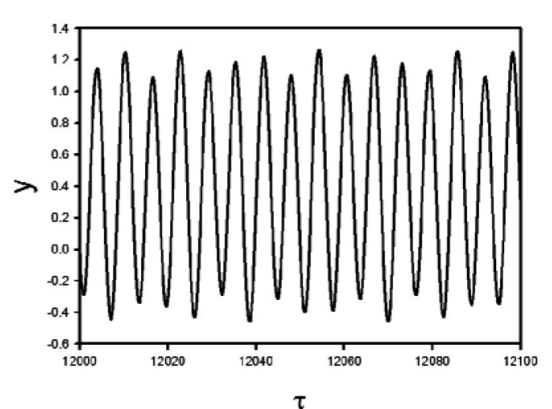

(a)

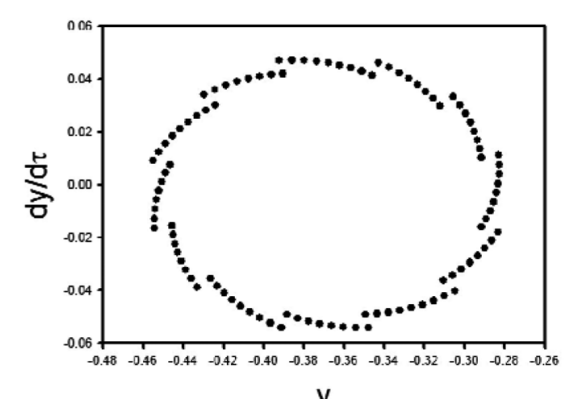

(c)

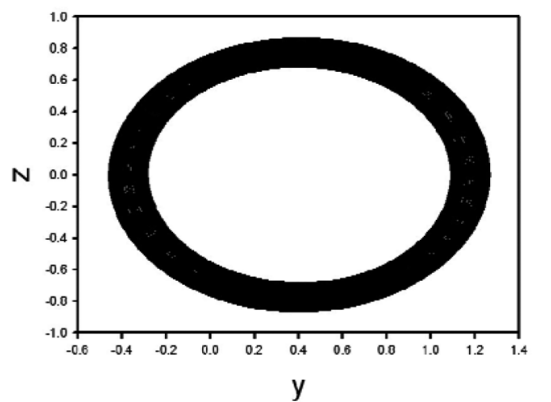

(b)

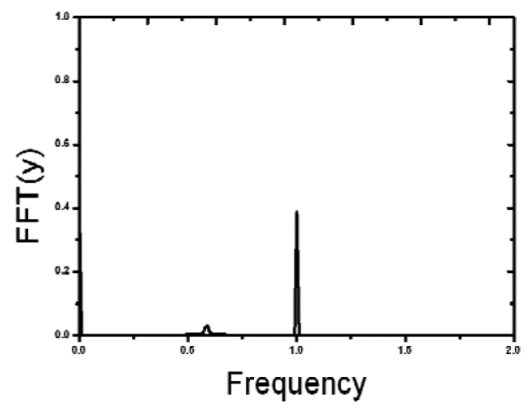

(d)

Fig. 15. Dynamic characteristic of the y-displacement of the mid-span point with $\gamma_{m}=100 \mathrm{~T}^{2} \mathrm{~m} / \mathrm{H}$. (a) Time history; (b) Orbital trajectory; (c) Poincaré section; (d) FFT.

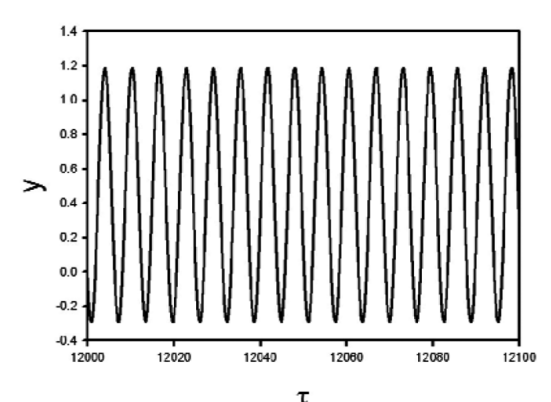

(a)

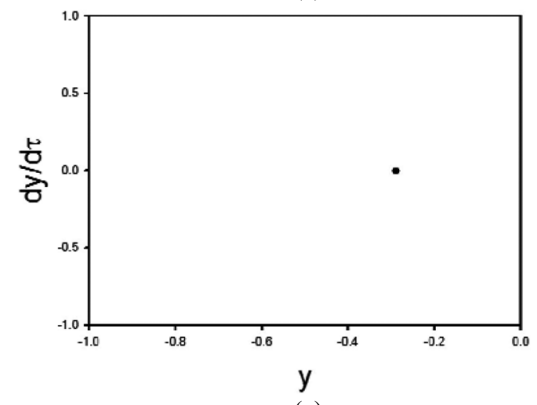

(c)

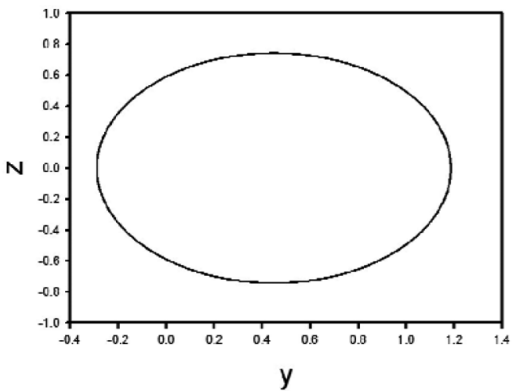

(b)

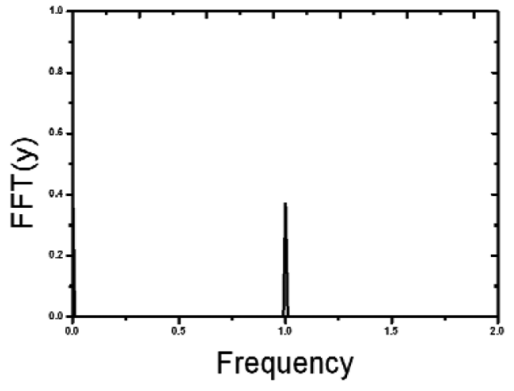

(d)

Fig. 16. Dynamic characteristic of the y-displacement of the mid-span point with $\gamma_{m}=2000 \mathrm{~T}^{2} \mathrm{~m} / \mathrm{H}$. (a) Time history; (b) Orbital trajectory; (c) Poincaré section; (d) FFT. 


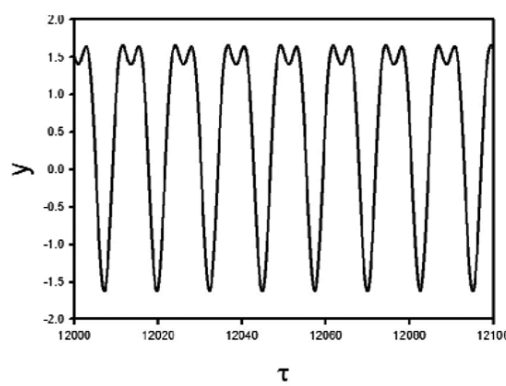

(a)

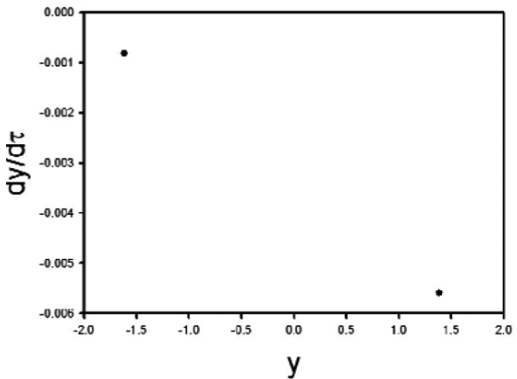

(c)

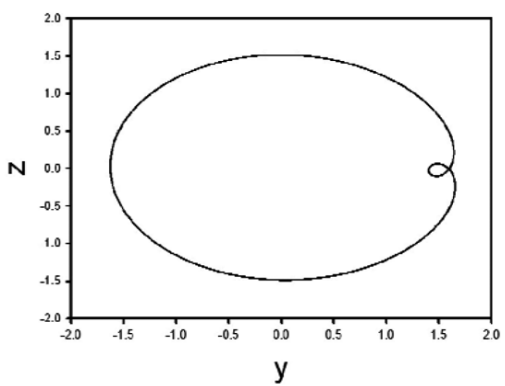

(b)

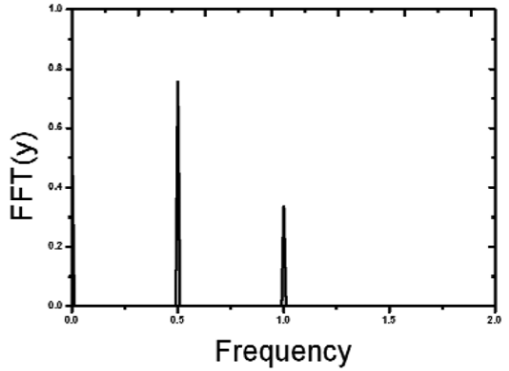

(d)

Fig. 17. Dynamic characteristic of the y-displacement of the mid-span point with $\gamma_{m}=4000 \mathrm{~T}^{2} \mathrm{~m} / \mathrm{H}$. (a) Time history; (b) Orbital trajectory; (c) Poincaré section; (d) FFT.

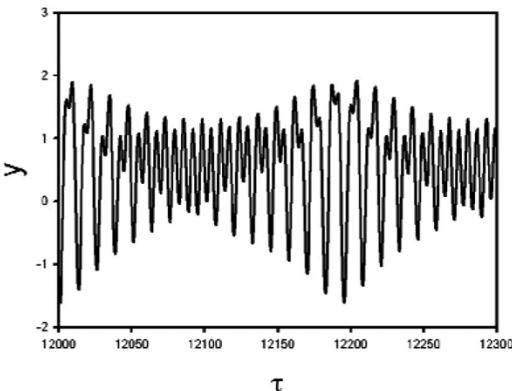

(a)

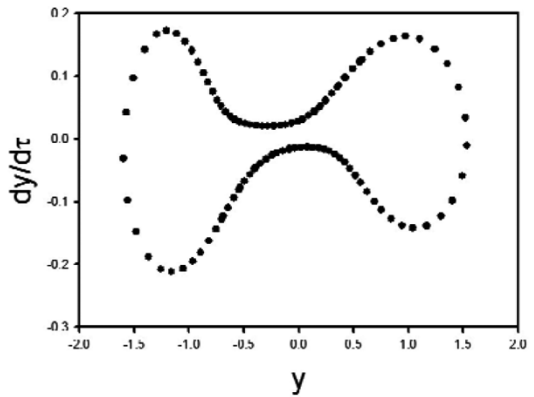

(c)

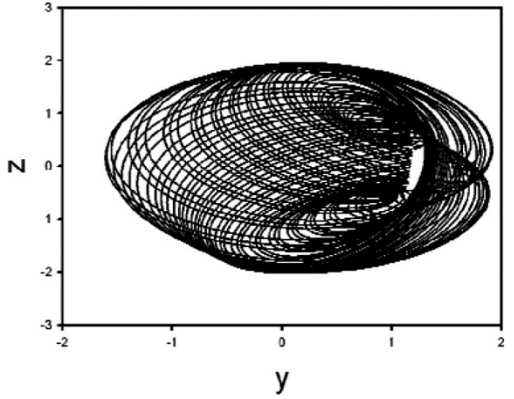

(b)

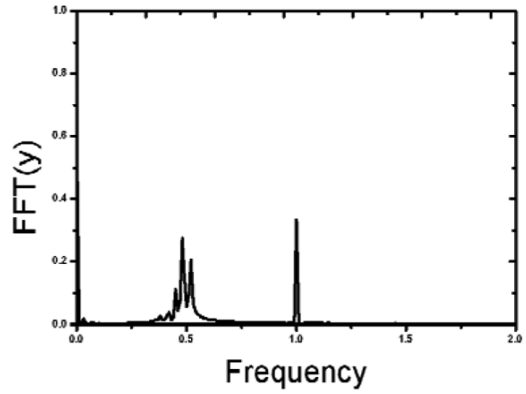

(d)

Fig. 18. Dynamic characteristic of the y-displacement of the mid-span point with $\gamma_{m}=4500 \mathrm{~T}^{2} \mathrm{~m} / \mathrm{H}$. (a) Time history; (b) Orbital trajectory; (c) Poincaré section; (d) FFT. 


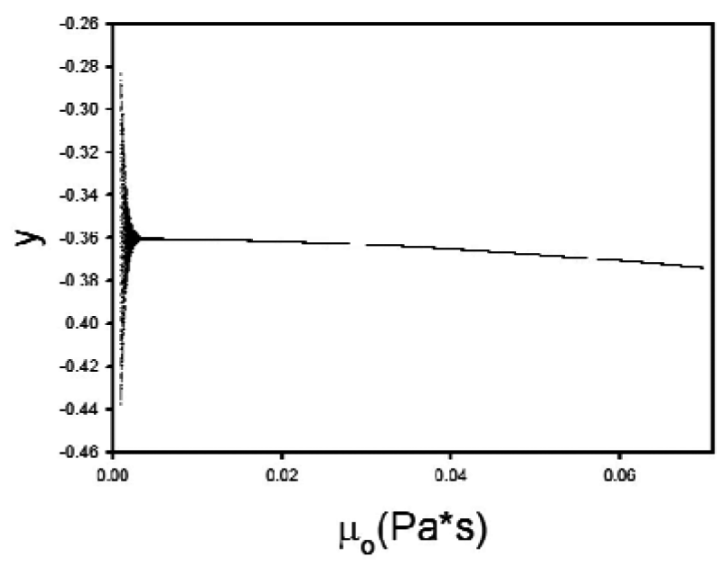

(a)

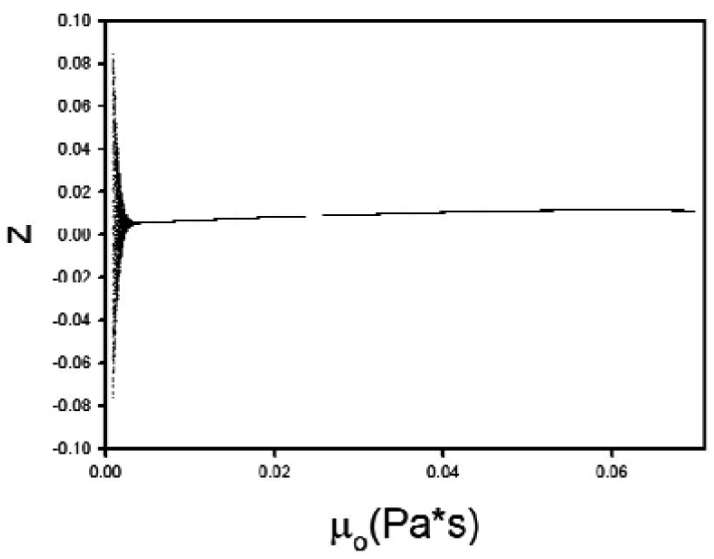

(b)

Fig. 19. The bifurcation diagram of displacements at the mid-span point versus oil viscosity. (a) $y$-displacement; (b) $z$-displacement. $D=0.25 \mathrm{~m}$, $\omega=600 \mathrm{rad} / \mathrm{s}, \gamma_{m}=300 \mathrm{~T}^{2} \mathrm{~m} / \mathrm{H}, \rho_{s}=7800 \mathrm{~kg} / \mathrm{m}^{3}, E=206 \mathrm{GPa}, C=0.001 D, C_{e}=D / 1600, L=3 \mathrm{~m}, L_{b}=D / 4, e_{\bar{m}}=0.0001 \mathrm{~m}$, $x_{a}=0.08, x_{b}=0.92$.

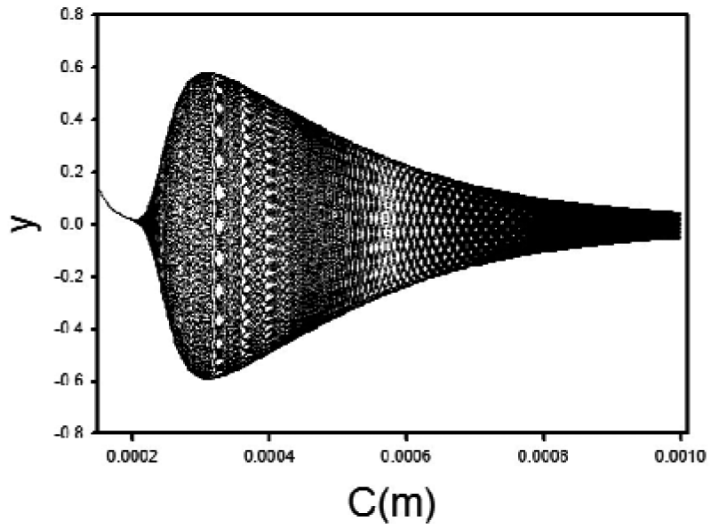

(a)

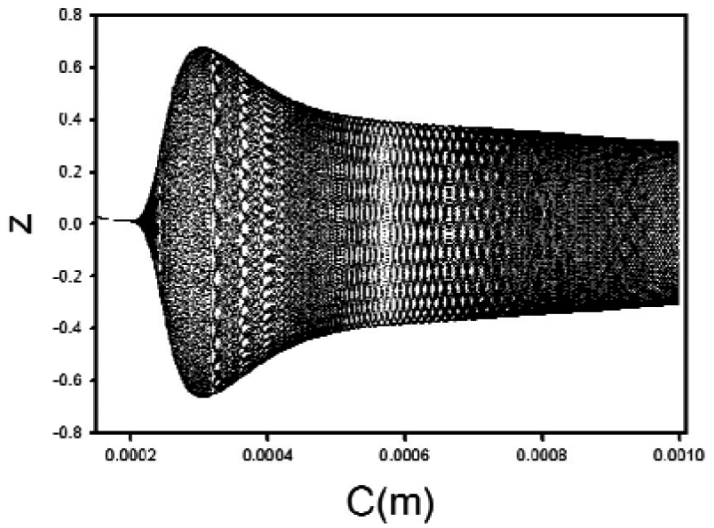

(b)

Fig. 20. The bifurcation diagram of displacements at the mid-span point versus mean clearance of bearing. (a) $y$-displacement; (b) $z$-displacement. $D=0.2 \mathrm{~m}, \omega=600 \mathrm{rad} / \mathrm{s}, \gamma_{m}=300 \mathrm{~T}^{2} \mathrm{~m} / \mathrm{H}, \rho_{s}=7800 \mathrm{~kg} / \mathrm{m}^{3}, E=206 \mathrm{GPa}, C_{e}=D / 1600, L=3 \mathrm{~m}, L_{b}=D / 4, e_{\bar{m}}=0.0001 \mathrm{~m}, x_{a}=$ $0.08, x_{b}=0.92, \mu_{o}=0.001 \mathrm{~Pa} \cdot \mathrm{s}$.

\subsection{Influence of bearing parameters}

In this section we investigate how dynamic characteristic of the system is influenced by key parameters of the bearing including viscosity of oil, mean clearance, and diameter of the bearing. Let $D=0.25 \mathrm{~m}, \omega=600 \mathrm{rad} / \mathrm{s}$, $\gamma_{m}=300 \mathrm{~T}^{2} \mathrm{~m} / \mathrm{H}$ and $C_{e}=D / 1600$. The bifurcation diagrams of $y$ - and $z$-displacements of the mid-span point of the shaft are illustrated in Figs 19(a) and 19(b) with the viscosity varying between $0.001 \mathrm{~Pa} \cdot \mathrm{s}$ and $0.07 \mathrm{~Pa} \cdot \mathrm{s}$. It can be concluded that large viscosity is beneficial for stable operation of the rotor system.

The second adjustable parameter is the mean radial clearance of the bearing which is generally a small quantity of $O\left(10^{-3}\right)$ compared to the diameter of the bearing. In this analysis, we choose $D=0.2 \mathrm{~m}, \omega=600 \mathrm{rad} / \mathrm{s}, \gamma_{m}=$ $300 \mathrm{~T}^{2} \mathrm{~m} / \mathrm{H}, \mu_{0}=0.001 \mathrm{~Pa} \cdot \mathrm{s}$ and $C_{e}=D / 1600$. In Fig. 20, it is demonstrated that the motion is period-1 for clearance $0.00015<C<0.0002 \mathrm{~m}$. The motion becomes quasi-periodic with $0.0002<C<0.001 \mathrm{~m}$. Therefore, a range of clearance $C=D / 1000 \sim D / 1300$ will most likely to secure safe operation of the rotor system.

Another influential parameter related to the bearings is the diameter of journal which is assumed equal to the diameter of the shaft for convenience. Let $\omega=500 \mathrm{rad} / \mathrm{s}, \gamma_{m}=500 \mathrm{~T}^{2} \mathrm{~m} / \mathrm{H}, \mu_{0}=0.001 \mathrm{~Pa} \cdot \mathrm{s}$ and $C_{e}=D / 1600$. 


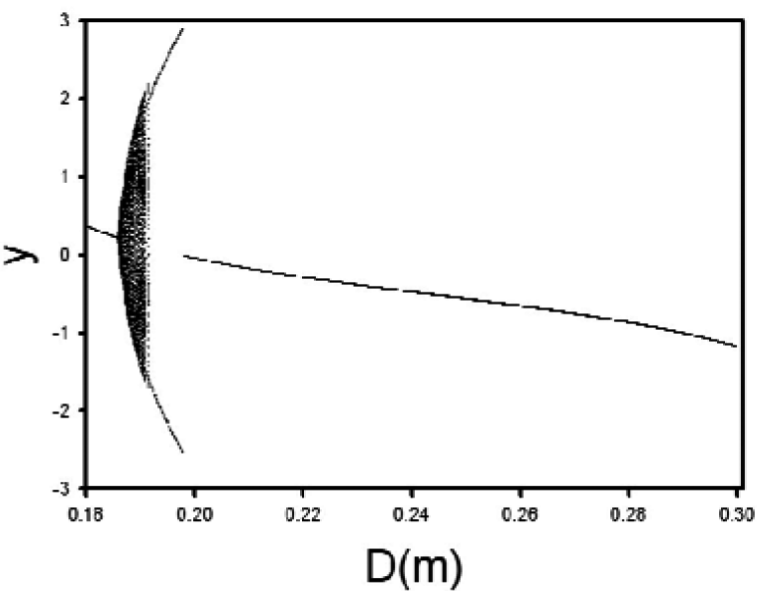

(a)

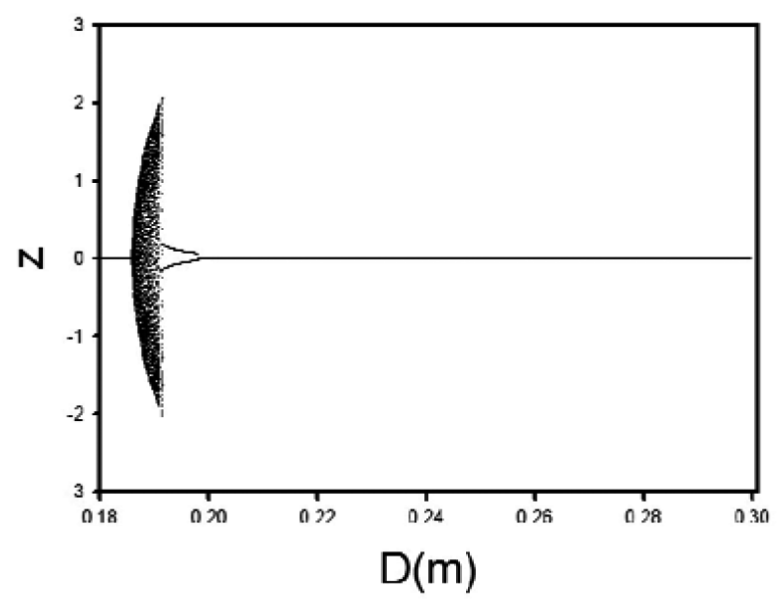

(b)

Fig. 21. The bifurcation diagram of displacements at the mid-span point versus diameter of journal. (a) $y$-displacement; (b) $z$-displacement. $\omega=$ $500 \mathrm{rad} / \mathrm{s}, \gamma_{m}=500 \mathrm{~T}^{2} \mathrm{~m} / \mathrm{H}, \rho_{s}=7800 \mathrm{~kg} / \mathrm{m}^{3}, E=206 \mathrm{GPa}, C=0.001 D, C_{e}=D / 1600, L=3 \mathrm{~m}, L_{b}=D / 4, e_{\bar{m}}=0.0001 \mathrm{~m}, x_{a}=$ $0.08, x_{b}=0.92, \mu_{o}=0.001 \mathrm{~Pa} \cdot \mathrm{s}$.

Figures 21(a) and 21(b) show that the motion is unstable and the rotor cannot be operated safely with the diameter less than $0.18 \mathrm{~m}$. Period-1 motion can be observed for larger diameter $0.18 \mathrm{~m}<D<0.186 \mathrm{~m}$. The motion becomes quasi-periodic with $0.186 \mathrm{~m}<D<0.191 \mathrm{~m}$ and period-2 with $0.191 \mathrm{~m}<D<0.198 \mathrm{~m}$. Afterwards, the motion returns period-1 with larger journal diameter.

\section{Conclusions}

The nonlinear vibration of a continuum rotor supported by two oil-film bearings and excited by longitudinally distributed electromagnetic load is investigated in this paper. The governing equation of motion of the rotor-bearing system is derived and discretized as a group of ordinary differential equations. The stability of equilibrium position of the rotor is analyzed and occurrence of the Andronov-Hopf bifurcation in the parameter domain is pointed out. The steady response of the system is presented for the primary resonance using the method of averaging. The transient displacements of the rotor are numerically solved through the Runge-Kutta method. Multiple plots are provided to show bifurcation of the displacements with varying rotation speed, electromagnetic parameter and parameters of bearing. Diagrams of time history, orbital trajectory, the Poincaré section and the fast Fourier transformation of the transverse vibration are presented to show complicated dynamic characteristics of the system with response of period-1, period-2 and various quasi-periodic motions.

\section{Acknowledgements}

The authors are grateful for fundings from the Exploration Projects on Nuclear Pumps of Dalian University of Technology, the National Science Foundation of China (10721062) and the State Key Development Program for Basic Research of China (Projects 2009CB724300 and 2011CB706504).

\section{Appendix A: Expressions of integration constants of Eq. (8)}

$$
H_{1}=\int_{0}^{1}(\sin \pi x)^{2} d x,
$$




$$
\begin{aligned}
& H_{2}=\int_{0}^{1} \frac{d^{4}(\sin \pi x)}{d x^{4}} \cdot \sin \pi x d x, \\
& H_{3}=\int_{0}^{1}(\sin \pi x)^{4} d x \\
& H_{4}=\int_{0}^{1}(\sin \pi x)^{2} \cdot(\sin 2 \pi x)^{2} d x, \\
& H_{5}=\int_{0}^{1} \sin \pi x d x, \\
& H_{6}=\int_{0}^{1}\left(\delta\left(x-x_{a}\right)+\delta\left(x-x_{b}\right)\right) \cdot \sin \pi x d x, \\
& H_{7}=\int_{0}^{1}\left(\delta\left(x-x_{a}\right)+\delta\left(x-x_{b}\right)\right) \cdot(\sin \pi x)^{4} d x, \\
& L_{6}=\int_{0}^{1}\left(\delta\left(x-x_{a}\right)+\delta\left(x-x_{b}\right)\right) \cdot(\sin \pi x)^{2} \cdot(\sin 2 \pi x)^{2} d x, \\
& H_{8}=\int_{0}^{1}\left(\delta\left(x-x_{a}\right)+\delta\left(x-x_{b}\right)\right) \cdot(\sin \pi x)^{2} \cdot(\sin 2 \pi x)^{2} d x, \\
& L_{3}=\int_{0}^{1}(\sin \pi x)^{2} \cdot(\sin 2 \pi x)^{2} d x, \\
& L_{1}=\int_{0}^{1}(\sin 2 \pi x)^{2} d x, \\
& L_{2}=\int_{0}^{1} \frac{d^{4}(\sin 2 \pi x)}{d x^{4}} \cdot \sin 2 \pi x d x, \\
& L_{0}
\end{aligned}
$$

\section{References}

[1] E.W. Summers, Vibration in 2-pole induction motors related to slip frequency, Power Apparatus and Systems, Part III. Transactions of the American Institute of Electrical Engineers 74(1) (1955), 69-72.

[2] R. Belmans, A. Vandenput and W. Geysen, Calculation of the flux density and the unbalanced pull in two pole induction machines, Electrical Engineering 70(3) (1987), 151-161.

[3] A.C. Smith and D.G. Dorrell, Calculation and measurement of unbalanced magnetic pull in cage induction motors with eccentric rotors. Part 1: Analytical model, IEE Proceedings-electric Power Applications 143(3) (1996), 193-201.

[4] D.G. Dorrell and A.C. Smith, Calculation and measurement of unbalanced magnetic pull in cage induction motors with eccentric rotors. Part 2: Experimental investigation, IEE Proceedings-electric Power Applications 143(3) (1996), 202-210.

[5] C. Bi, Z.J. Liu and T.S. Low, Effects of unbalanced magnetic pull in spindle motors, IEEE Transactions on Magnetics 33(5) (1997), 4080-4082. 
[6] N.L.P. Lundström and J.O. Aidanpää, Dynamic consequences of electromagnetic pull due to deviations in generator shape, Journal of Sound and Vibration 301(1-2) (2007), 207-225.

[7] R. Belmans, A. Vandenput and W. Geysen, Influence of unbalanced magnetic pull on the radial stability of flexible-shaft induction machines, IEE Proceedings-B Electric Power Applications 134(2) (1987), 101-109.

[8] D. Guo, F. Chu and D. Chen, The unbalanced magnetic pull and its effects on vibration in a three-phase generator with eccentric rotor, Journal of Sound and Vibration 254(2) (2002), 297-312.

[9] P. Pennacchi, Nonlinear effects due to electromechanical interaction in generators with smooth poles, Nonlinear Dynamics 57(4) (2009), $607-622$.

[10] Y.F. Wang, L.H. Huang and Y. Li, Nonlinear vibration and stability of a jeffcott rotor under unbalanced magnetic excitation, International Journal of Nonlinear Sciences and Numerical Simulation 8(3) (2007), 375-384.

[11] H.Y. Luo and Y.F. Wang, Stability and Bifurcation of a Continuum Rotor with Transverse Electromagnetic and Bearing Excitations, Applied Mechanics and Materials 105-107 (2012), 439-443.

[12] A. Muszynska, Rotordynamcis, Taylor \& Francis, Boca Raton, (2005).

[13] Y. Ishida, Nonlinear vibration and chaos in rotordynamics, JSME International Journal Series C-Dynamics Control Robotics Design and Manufacturing 37(2) (1994), 237-245.

[14] R.D. Brown, P. Addison and A.H.C. Chan, Chaos in the unbalance response of journal bearings, Nonlinear Dynamics 5(4) (1994), 421-432.

[15] G. Adiletta, A.R. Guido and C. Rossi, Chaotic motions of a rigid rotor in short journal bearing, Nonlinear Dynamics 10(3) (1996), $251-269$.

[16] Y.B. Kim and S.T. Noah, Quasi-periodic response and stability analysis for a non-linear Jeffcott rotor, Journal of Sound and Vibration 190(2) (1996), 239-253.

[17] J.H. He, Exact resonances of nonlinear vibration of rotor-bearings system without small parameter, Mechanics Research Communications 27(4) (2000), 451-456.

[18] Q. Ding, J.E. Cooper and A.Y.T. Leung, Hopf bifurcation analysis of a rotor/seal system, Journal of Sound and Vibration 252(5) (2002), $817-833$.

[19] Y.F. Wang and X.Y. Wang, Nonlinear vibration analysis for a Jeffcott rotor with seal and air-film bearing excitation, Mathematical Problems in Engineering 2010, 1-14.

[20] A. Muszynska, Improvements in lightly loaded rotor/bearing and rotor/seal models, Journal of Vibration Acoustics Stress and Reliability in Design-Transactions of the ASME 110(2) (1988), 129-136.

[21] M.F.A. Azeez and A.F. Vakakis, Numerical and experimental analysis of a continuous overhung rotor undergoing vibro-impacts, International Journal of Non-Linear Mechanics 34(3) (1998), 415-435.

[22] F. Oncescu, A.A. Lakis and G. Ostiguy, Investigation of the stability and steady state response of asymmetric Rotors, using finite element formulation, Journal of Sound Vibration 245(2) (2001), 303-328.

[23] J.P. Jing, G. Meng, Y. Sun and S.B. Xia, On the oil-whipping of a rotor-bearing system by a continuum model, Applied Mathematical Modelling 29(5) (2005), 461-475.

[24] S.A.A. Hosseini and S.E. Khadem, Combination resonances in a rotating shaft, Mechanism and Machine Theory 44(8) (2009), $1535-1547$.

[25] L. Meirovitch, Fundamentals of Vibrations, McGRAW-HILL, Singapore (2001).

[26] F.L. Chu and Z.S. Zhang, Periodic, quasi-periodic and chaotic vibrations of a rub-impact rotor system supported on oil film bearings, International Journal of Engineering Science 35(10-11) (1997), 963-973. 

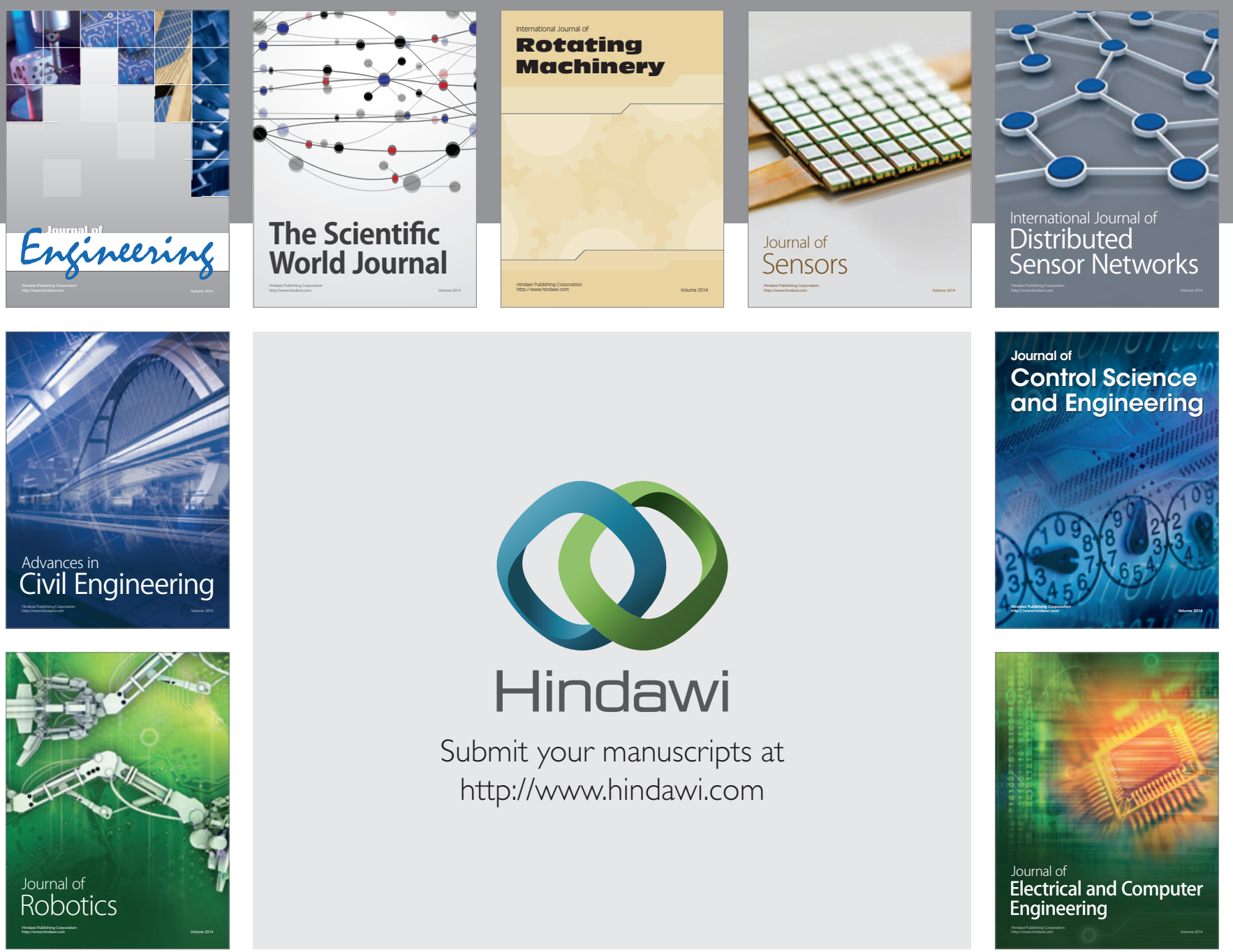

Submit your manuscripts at

http://www.hindawi.com
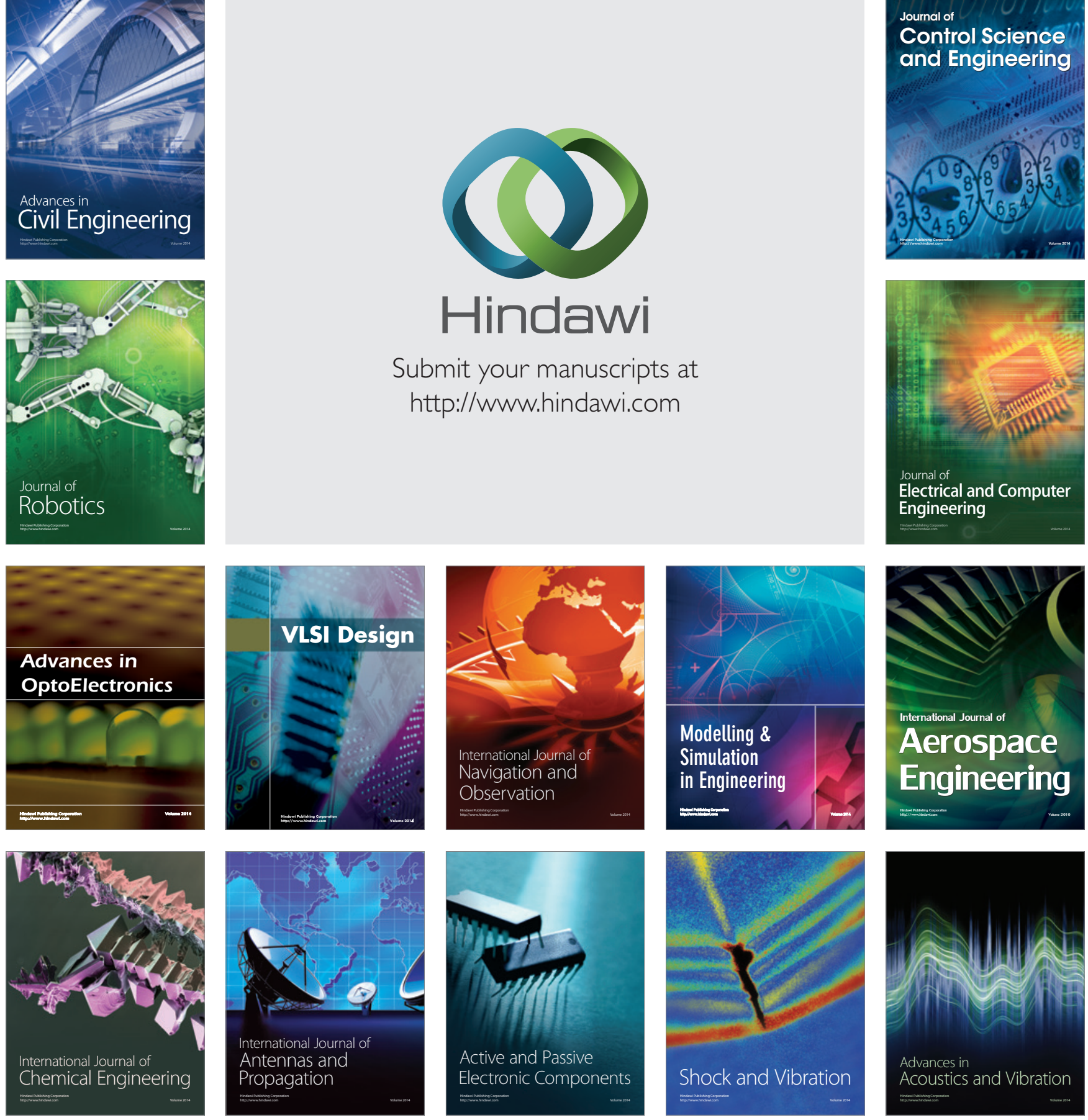